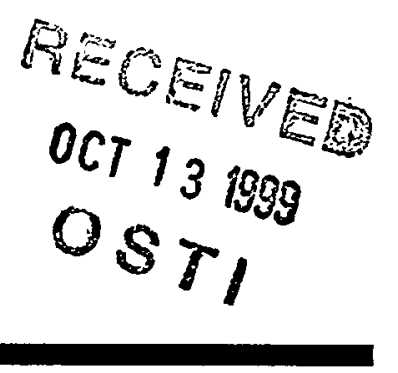

\title{
Recovery of Flexible Polyurethane Foam from Shredder Residue
}

Energy Systems Division Argonne National Laboratory

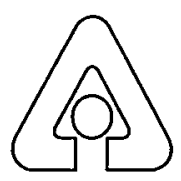

Operated by The University of Chicago, under Contract W-31-109-Eng-38, for the

United States Department of Energy 


\section{Argonne National Laboratory}

Argonne National Laboratory, with facilities in the states of Illinois and Idaho, is owned by the United States Government, and operated by the University of Chicago under the provisions of a contract with the Department of Energy.

This technical memo is a product of Argonne's Energy Systems (ES) Division. For information on the division's scientific and engineering activities, contact:

Director, Energy Systems Division

Argonne National Laboratory

Argonne, Illinois 60439-4815

Telephone (630) 252-3724

Presented in this technical memo are preliminary results of ongoing work or work that is more limited in scope and depth than that described in formal reports issued by the ES Division.

Publishing support services were provided by Argonne's Information and Publishing Division (for more information, see IPD's home page: http://www.jpd.anl.gov/).

\section{Disclaimer}

This report was prepared as an account of work sponsored by an agency of the United States Government. Neither the United States Government nor any agency thereof, nor any of their employees, makes any warranty, express or implied, or assumes any legal liability or responsibility for the accuracy, completeness, or usefulness of any information, apparatus, product, or process disclosed, or represents that its use would not infringe privately owned rights. Reference herein to any specific commercial product, process, or service by trade name, trademark, manufacturer, or otherwise, does not necessarily constitute or imply its endorsement, recommendation, or favoring by the United States Government or any agency thereof. The views and opinions of authors expressed herein do not necessarily state or reflect those of the United States Government or any agency thereof.

Reproduced directly from the best available copy.

Available to $D O E$ and DOE contractors from the Office of Scientific and Technical Information, P.O. Box 62, Oak Ridge, TN 37831; prices available from (423) 576-8401.

Available to the public from the National Technical Information Service, U.S. Department of Commerce, 5285 Port Royal Road, Springfield, VA 22161. 


\section{DISCLAIMER}

Portions of this document may be illegible in electronic image products. Images are produced from the best available original document. 


\section{.}




\section{Recovery of Flexible Polyurethane Foam from Shredder Residue}

B.J. Jody and E.J. Daniels

Energy Systems Division

Argonne National Laboratory, 9700 South Cass Avenue, Argonne, lllinois 60439

June 1999

Work sponsored by the U.S. Department of Energy, Assistant Secretary for Energy Efficiency and Renewable Energy, Office of Industrial Technologies, under contract W-31-109-Eng-38. 



\section{Contents}

Notation and Definitions..................................................................................... vi

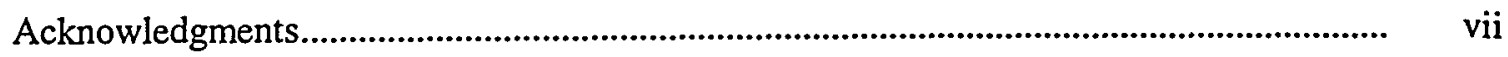

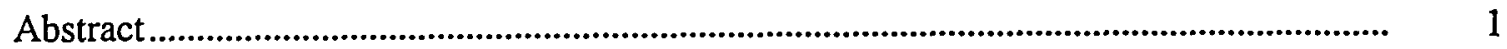

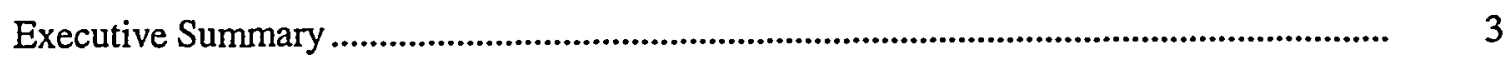

1 Introduction and Background .............................................................................. 5

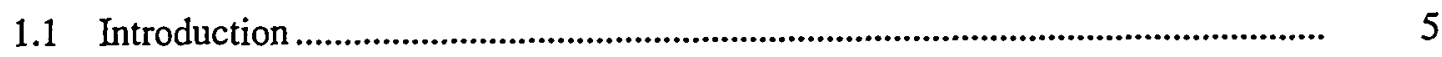

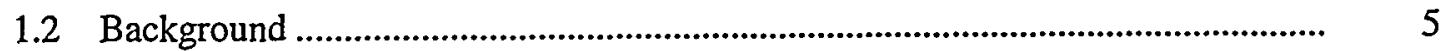

1.3 Options for Handling Shredder Residue ............................................................... 5

1.3.1 Characterization of Shredder Residue .................................................... 7

1.3.2 Bulk Separation of Shredder Residue........................................................ 7

1.3.3 Use of Shredder Residue Fines............................................................... 7

1.3.4 Selective Recovery of Thermoplastics from Shredder Residue .................. 8

1.3.5 Thermochemical Conversion of Shredder Residue .................................. 8

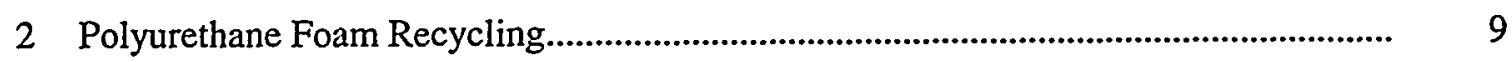

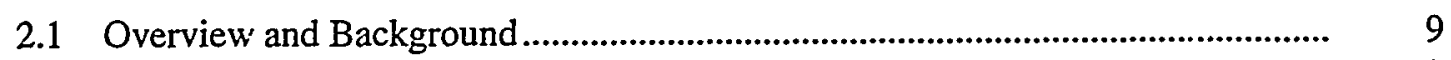

2.2 Process Research and Development....................................................................... 9

2.2.1 Bench-Scale Proof-of-Concept Research ....................................................... 9

2.2.2 Batch Process Pilot-Scale Testing ................................................................ 11

2.2.3 Continuous Process Pilot-Scale Testing ........................................................ 11

2.3 Polyurethane Foam Recovery Process ................................................................... 12

2.3.1 Unit Operation 1: ASR Screening and PUF Recovery ............................... 12

2.3.2 Unit Operation 2: PUF Sizing...................................................................... 13

2.3.3 Unit Operations 3, 4, and 5: Washing, Rinsing, and Drying ........................ 13

2.3.4 Unit Operation 6: Baling .......................................................................... 15

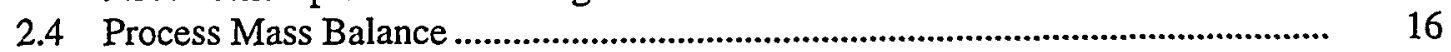

2.5 Disposition of the Process Reject/Waste Streams ................................................ 16

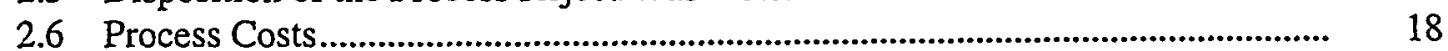

2.7 Market Potential for Recycled Foam ................................................................. 19

2.8 Market Opportunity/Supply Side ........................................................................ 19

2.9 Recycled Foam Product Testing .......................................................................... 20

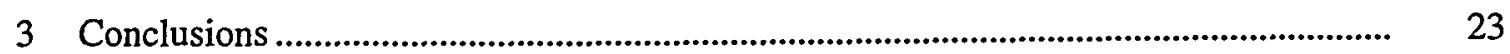

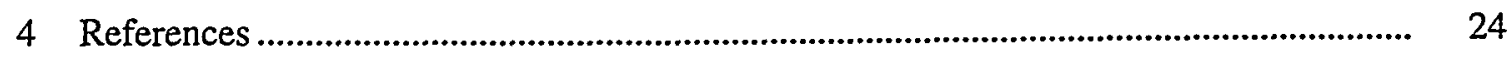




\section{Contents (Cont.)}

5 Annotated Bibliography of Argonne Shredder Residue Research................................ 26

Appendix A: The Argonne Bulk Mechanical Separation Pilot Plant..................................... A-1

Appendix B: Use of Shredder Residue's Inorganic Fines .................................................... B-1

Appendix C: Selective Recovery of Thermoplastics by Using Froth Flotation....................... C-1

Appendix D: Recycled Foam Material Information Sheet ................................................ D-1

Appendix E: U.S. Patent: Efficient Continuous Dryer for Flexible Polyurethane Foam and Cleaning Apparatus .......................................................................... E-1

Appendix F: Chemistry of the Waste Streams .................................................................. F-1

Figures

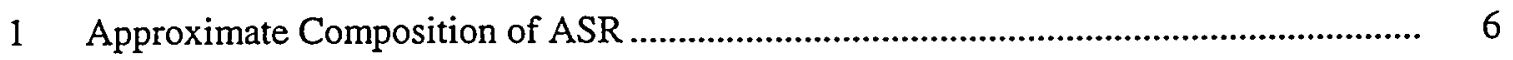

2 Polyurethane Foam as Recovered from Shredder Residue and after Cleaning .................................................................................................... 10

3 Block Flow Diagram of the PUF Recovery Process ...................................................... 13

$4 \quad$ Wash, Rinse, and Dry Continuous Equipment............................................................ 14

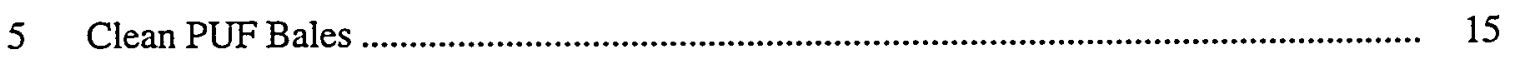

6 Process Flow Sheet with Material Balance .............................................................. 17

7 U.S. Foam Consumption by the Transportation and Furniture Industries ...................... 19

8 Distribution of Shredders and Foam Recovery Potential as a Function of the Annual Recovery Potential per Plant Site ........................................................................ 20

9 Noise Reduction of Automotive Carpet Underlayment ................................................ 21

10 Recovered Foam from ASR Made into Carpet Padding ............................................. 22

A.1 Argonne Materials Separation Facility ............................................................... A-2

C.1 Growth in Major Automotive Plastics ..................................................................... C-4 


\section{Tables}

1 Variability of ASR Composition ..............................................................................

2 PUF Recovery Process Costs .................................................................................... 18

B.1 Elemental Metal Analysis of the Total Fines and the Magnetic Fraction of the Fines.......................................................................................... B-1

B.2 Results of the TCLP Analysis of Magnetic Fines Derived from ASR ............................. B-2

C.1 Values and Quantities of the Major Plastics in ASR ...................................................... C-1

F.1 Results of the TCLP Analysis of the Solid Waste Generated by the Continuous Foam Cleaning Process

F.2 Results of the TCLP Analysis of the Oil Waste Generated by the Continuous Foam Cleaning Process

F.3 Results of the TCLP Analysis of the Waste Water Generated by the Continuous Foam Cleaning Process. 


\section{Notation and Definitions}

ABS - Acrylonitrile-butadiene-styrene.

APC - American Plastics Council. A membership organization that consists of about 25 major plastics producers.

ASR - Auto Shredder Residue. The reject material that is landfilled after processing scrap (such as obsolete cars and appliances) for recovery of metals.

ARA - Automotive Recyclers' Association. The trade association that represents about 12,000 auto dismantlers - companies that typically recycle cars for used parts.

Fines - Materials in ASR less than 0.25 in. Typically fines consist of metal oxides (e.g., rust), glass, dirt, wood, and small particles of plastics, rubber, and other materials.

ISRI - Institute of Scrap Recycling Industries. The trade association that represents scrap material recyclers including about 200 shredder operators who recover recyclable metals and materials from obsolete cars.

Obsolete Scrap - Post-consumer scrap, such as obsolete cars, appliances, and other household items.

Polyolefins - Polyethylene and polypropylene.

Prompt Scrap - Scrap generated during the manufacturing process; industrial scrap.

PVC - Polyvinyl chloride.

Rebond - The generic term that refers to the process whereby scrap foam is chemically rebonded to produce recycled foam products.

VRP - Vehicle Recycling Partnership. An organization formed by General Motors, Ford, and DaimlerChrysler to promote the recycling of obsolete automobiles. 


\section{Acknowledgments}

This work was sponsored by the U.S. Department of Energy, Assistant Secretary for Energy Efficiency and Renewable Energy, Office of Industrial Technologies, under contract W-31-109-Eng-38. 
$\Delta$ 


\section{Abstract}

Argonne National Laboratory has developed a patented, continuous process for the recovery of flexible polyurethane foam (PUF) from auto shredder residue (ASR). To test the process, Argonne researchers conceived of, designed, and built a continuous foam washing and drying system that was pilot-tested at a shredder facility for six months. Economic analysis of the process, using manufacturers' quotes and operating data from Argonne's pilot plant, indicates a payback of less than two years for a plant producing about 1,000 ton/yr of foam. Samples of clean foam were shipped to three major foam reprocessors; all three indicated that the quality of the PUF recovered by the Argonne process met their requirements. Tests of the recovered foam by an independent testing laboratory showed that the recycled foam met the specifications for several automotive applications, including carpet padding, headliner, and sound-suppression support materials. Recovery of foam reduces the mass and the volume of material going to the landfill by about $5 \%$ and $30 \%$, respectively. Annually, recovery will save about $1.2 \times 10^{12} \mathrm{Btu}$ of energy, cut the amount of solid waste being landfilled by about 150,000 tons, and eliminate the emission of about 250 tons of volatile organic compounds (VOCs) into the air. 
$\Delta-$ 


\section{Executive Summary}

Argonne National Laboratory staff have developed a patented process for the recovery of flexible polyurethane foam (PUF) from auto shredder residue (ASR). This process evolved from bench-scale research through continuous pilot operation. The overall continuous process consists of six basic unit operations: (1) foam separation and screening, (2) sizing, (3) washing, (4) rinsing, (5) drying, and (6) baling. A preliminary design for a full-scale plant was also developed on the basis of the results obtained from the pilot-plant operation, and an economic analysis of the process was completed. The expected payback is less than $2 \mathrm{yr}$.

Argonne has conceived of, designed, and built a continuous foam washing and drying pilot-plant system with a design capacity of $100 \mathrm{lb}$ of foam per hour. The system was pilottested at a shredder facility for 6 months. The system was operated continuously in staged campaigns with no process upsets. During the pilot operation, over 3.5 tons of clean foam was produced. The necessary engineering and operations data for designing a commercial-scale plant and confirming the process economics were also produced during the pilot-plant operations. Economic analysis of the process, using manufacturers' quotes and operating data from the pilot plant, indicates a payback of less than two years for a plant producing about 1,000 tons per year of foam. This amount of PUF is estimated to be recoverable from a shredder processing $60 \mathrm{cars} / \mathrm{h}$ in a single shift.

Samples of clean foam were shipped to three major foam reprocessors: Carpenter Co., Vita Foam, and Woodbridge Group. All three indicated that the quality of the PUF recovered by the Argonne process met their requirements.

Foam recovered by the process was also used for making carpet padding that was installed and tested in three 1997 vehicles: Jeep Cherokee, Saturn, and the Mercury Villager. The results indicated that, for this application, the recycled foam performed as well as conventional materials. Samples of the recovered foam were also sent to an independent testing laboratory to determine if the foam meets the specifications of some large automotive applications. The test results showed that the recycled foam met the specifications for several automotive applications, including carpet padding, headliner, and sound-suppression support materials.

The 185 or so U.S. shredders who shred over 10 million vehicles annually (in addition to other metals-containing items) produce between 3 and 5 million tons a year of ASR, essentially all of which is landfilled. The ASR contains about 150,000 tons of recoverable polyurethane foam, the value of which is about $\$ 90$ million $(\$ 0.30 / \mathrm{lb})$. Recovery of the foam reduces the mass and the volume of material going to the landfill by about $5 \%$ and $30 \%$, respectively. Annually, recovery will save about $1.2 \times 10^{12}$ Btu of energy, cut the amount of solid waste being landfilled by about 150,000 tons, and eliminate the emission of about 250 tons of VOCs into the air. 
A 


\section{Section 1 \\ Introduction and Background}

\subsection{Introduction}

Argonne National Laboratory, in collaboration with the Vehicle Recycling Partnership, the American Plastics Council, the Institute of Scrap Recycling Industries, and others, has been developing technology for cost-effectively recovering recyclable materials from a reject material commonly referred to as auto shredder residue (ASR). In this project, Argonne's objective was to develop a process for cost-effectively recovering flexible polyurethane foam (PUF) from ASR for reuse in the foam rebond market.

\subsection{Background}

The scrap recycling industry processes obsolete automobiles and other metal-bearing consumer durables (such as home appliances) to recover metals for recycling. Because only the metals are recycled at present, the balance of the materials (such as plastics, glass, rubber, fiber, etc.) are landfilled.

Almost all obsolete cars are processed for recycling. Typically, obsolete cars are first processed by the dismantling industry, which recovers usable parts and components for resale and reuse. There are more than 12,000 auto dismantlers located throughout North America. After reusable parts are recovered, the balance of the car - referred to as the hulk - is shipped to one of about 185 auto shredders for further processing to recover metals. The auto hulk is shredded in large hammermills that rip the hulk into pieces generally not larger than about $10 \mathrm{in.}$ in any dimension. In a typical shredder, one auto hulk can be processed in about $60 \mathrm{~s}$. After shredding, the ferrous and nonferrous metals are recovered for reuse by the iron and steel industry and the nonferrous metals industries. In general, the balance of the material is then landfilled. Three to five million tons of residue are landfilled each year in North America. Similar quantities of shredder residue are landfilled in Europe and Asia.

Although the composition of shredder residue varies (depending on the types and relative quantities of source materials processed, as well as the specific technology employed by the shredder operator), the typical composition of the waste materials that are landfilled is $50 \%$ inert material, $25 \%$ plastics (including polyurethane foam), $10 \%$ moisture, and $15 \%$ other materials (Figure 1).

\subsection{Options for Handling Shredder Residue}

A wide range of research has been conducted (and is still ongoing) to develop costeffective technologies for handling shredder residue. These technologies include the use of shredder residue as a fuel (i.e., waste to energy), chemical processing (e.g., pyrolysis, gasification, methanolysis, etc.) to produce fuels and chemicals from shredder residue, and the 


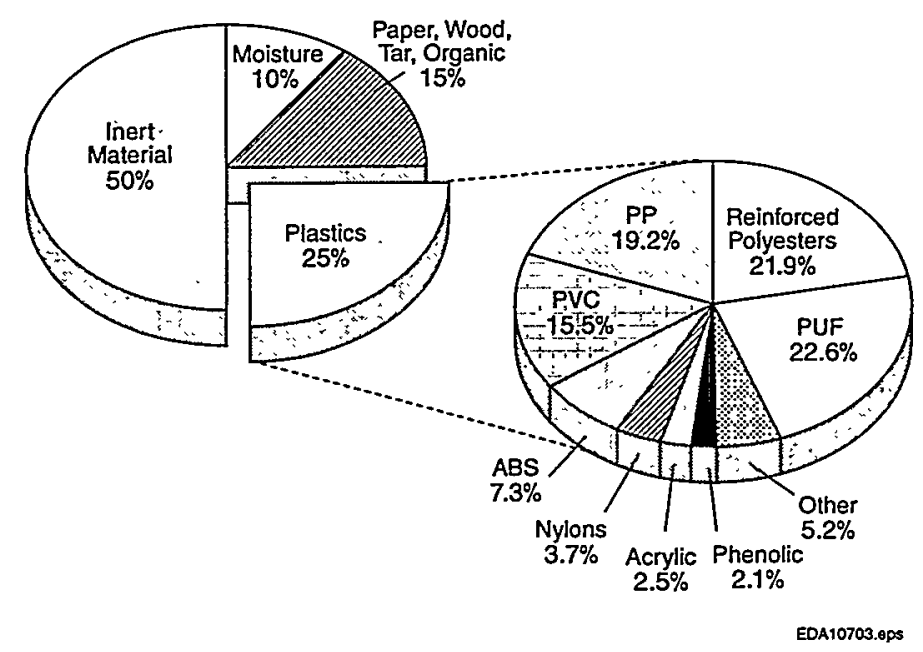

Figure 1 Approximate Composition of ASR (based on data for a 1981 model year vehicle*)

use of shredder residue as a bulk material (e.g., molding of shredder residue to produce parking stops.) Much of this work is discussed by Jody et al. [1,9].

As an alternative to handling shredder residue, research has also been conducted to recover materials from the dismantlers' facilities prior to shredding. In gentral, dismantling for materials recovery is not cost-effective, primarily because (1) dismantling for materials recovery is labor-intensive and (2) only a small amount of material can be recovered at the more than 12,000 dismantling yards, hence transport costs for recovered materials are generally prohibitive. The Auto Recyclers Association, the Vehicle Recycling Partnership, and other organizations are undertaking ongoing research to develop techniques that could reduce these costs.

The focus of the research conducted at Argonne has been to develop processes for the extraction of recyclable materials (such as PUF and selected thermoplastics) from shredder residue for reuse as a substitute for virgin material. This approach is similar to the current practice of recovering scrap steel from obsolete cars and using it as raw material for the production of new steel.

Process development research conducted at Argonne for the recovery of materials from shredder residue is discussed in detail by Jody et al. [1-13] and by Arzoumanidis et al. [14] and by Brockmeier and McIntosh [15]. This research is briefly summarized below.

* Data, in part, from McCellan, T.R., 1983, Modern Plastics, 59(2):50-52. 


\subsubsection{Characterization of Shredder Residue}

Argonne's initial research focused significant effort on characterizing shredder residue to determine the variability in its composition. Samples from a number of shredders over the past $10 \mathrm{yr}$ have been collected and analyzed. Although there is some variability in the composition of shredder residue, the variability is more attributable to (1) the type of shredder system operated (i.e., wet vs. dry) and (2) the fraction of the feed material that is made up of vehicles than to any other factors. Table 1 lists the composition and variability of shredder residue.

Table 1 Variability of ASR Composition

\begin{tabular}{|llc|}
\hline \multicolumn{1}{|c|}{ Material } & $\begin{array}{c}\text { As Received } \\
\text { wt. } \%\end{array}$ & $\begin{array}{c}\text { Dry Basis } \\
\text { wt. } \%\end{array}$ \\
\hline Moisture & $15 \pm 10$ & \\
Inerts & $45 \pm 10$ & 53 \\
Plastics* & $25 \pm 5$ & 29 \\
Polyurethane Foam & $4 \pm 2$ & 5 \\
Automotive Fluids & $2 \pm 2$ & 2 \\
Other & $9 \pm 3$ & 11 \\
\hline
\end{tabular}

* Excluding polyurethane foam

\subsubsection{Bulk Separation of Shredder Residue}

The heterogeneity of shredder residue poses a problem; the complexity of the mixture, as well as the variation in the physical dimensions of the material, requires that a bulk separation process step be used to separate the "raw" shredder residue into more manageable fractions. To that end, research was conducted to develop a separation process for the bulk separation of shredder residue into three basic fractions: (1) polyurethane foam, which is very light and voluminous; (2) inorganic "fines," which are relatively dense and less than about 0.25 in. in any dimension; and (3) a middling fraction, or "plastics concentrate," that consists of the balance of the residue. Conventional bulk separation equipment was tested and found to be inadequate. Argonne developed and built a unique two-stage trommel to accomplish the bulk separation of shredder residue. A large-scale pilot plant with a 4,000-1b/h design capacity was built and has been used successfully to separate shredder residue from a number of shredding operations. The pilot plant, which is installed and operational at Argonne, is discussed in more detail in Appendix A.

\subsubsection{Use of Shredder Residue Fines}

The fines from shredder residue were found to constitute between $25 \%$ and $60 \%$ of the weight of shredder residue. The fines fraction, which is material less than $0.25 \mathrm{in}$. in any dimension, is essentially metal oxides, glass, dirt, and some plastics and fibers. Some work was conducted to further separate the fines fraction to produce a highly concentrated iron-oxide material that might be used as an alternative iron-oxide raw material for cement making. This work is discussed in Appendix B. 


\subsubsection{Selective Recovery of Thermoplastics from Shredder Residue}

Because a significant fraction of shredder residue is thermoplastics, and because the use of thermoplastics in cars is increasing, research is ongoing to selectively recover certain potentially high-volume, high-value thermoplastics for reuse as thermoplastics from shredder residue. Argonne's initial technical approach involved using solvents to extract individual plastics, including ABS, PVC, and a polyolefin blend, from shredder residue. Although the process was technically feasible in that high-purity resins could be extracted, process economics were prohibitive because the auxiliary system costs associated with the safety and environmental control equipment required when solvents are used were prohibitive relative to the quantity of specific thermoplastics that could be recovered per pound of shredder residue processed [1-6]. Research has since focused on the development of froth flotation technology for selectively recovering certain thermoplastics from shredder residue. This research is ongoing. A brief summary of this work to date is presented in Appendix C.

\subsubsection{Thermochemical Conversion of Shredder Residue}

Argonne also conducted a limited investigation into the feasibility of thermochemical conversion of shredder residue. Specifically investigated were controlled thermal extrusion to produce a synthetic particulate coal and catalytic pyrolysis to produce gaseous and liquid fuels. Again, although these processes are technically feasible, they are generally not cost-effective within the economic environment of North America (i.e., low landfill costs and low oil/energy costs relative to those costs in either Europe or Japan, countries in which this type of technology may be cost-effective)[14-16]. 


\section{Section 2 \\ Polyurethane Foam Recycling}

\subsection{Overview and Background}

As previously discussed, research indicated the need to separate shredder residue into three fractions, including one consisting of flexible polyurethane foam. Flexible polyurethane foam accounts for only about 5 weight per cent (wt\%) but 30 volume per cent of the shredder residue because of the bulkiness of the foam.

Even though the foam is only a small weight per cent of the shredder residue, it is a target recycle product from shredder residue because the market for recycled foam in North America is large. The foam rebond industry presently imports about 150 million $\mathrm{lb} / \mathrm{yr}$ of prompt scrap foam from Europe and Asia. The imported scrap supplements the more than 1.75 billion $\mathrm{lb}$ of virgin foam used to produce foam products, such as residential and commercial carpet padding, automotive carpet padding, car seat cushions, and other consumer and automotive products [17].

The viability of using foam recovered from shredder residue to supply the foam rebond market depends on two key factors: (1) development of a cost-effective process for recovering foam from shredder residue and (2) confirmation that the recovered foam meets the quality requirements of the market.

\subsection{Process Research and Development}

Development of the process for recovering polyurethane foam from shredder residue evolved from bench-scale research through continuous pilot-scale operation.

\subsubsection{Bench-Scale Proof-of-Concept Research}

Bench-scale research focused on two issues: (1) developing a mechanism for separating the foam from the shredder residue and (2) testing solutions for cleaning the foam once it was recovered.

Bench-scale research on separating shredder residue into three fractions resulted in the design of a multistep process, including a unique two-stage trommel for the separation of the foam from the balance of shredder residue (see Appendix A).

The foam recovered from shredder residue must be cleaned because the foam is an excellent absorber of automotive fluids, dirt, and grime (Figure 2). Up to $50 \%$ of the weight of the foam, as recovered from shredder residue, is due to moisture, entrained dirt, automotive fluids, metal dust, metal oxides, and glass. Bench-scale experiments were conducted by using several organic solvents, including hexane, acetone, perchloroethylene (conventional dry cleaning solvent), petroleum distillates, and a proprietary "green" solvent. The research was initially focused on organic solvents rather than aqueous-based solutions because the organic 
solvents could be redistilled and reused, thereby avoiding a wastewater problem that was anticipated with aqueous-based solutions. All of these solvents were able to clean the foam. The bench-scale tests consisted of squeezing the foam in the solvent several times. These solvents can be regenerated by distillation and reused with no apparent reduction in cleaning capability, except for the "green" solvent that gradually degraded upon thermal regeneration. Perchloroethylene was then selected for pilot testing because (1) commercial equipment using it is available, (2) it is not flammable, and (3) R\&D is under way in industry to find an environmentally benign replacement for it.

Small samples (less than $5 \mathrm{lb}$ ) of cleaned foam from the bench tests were provided to potential users of the foam; the users found the samples to be acceptable (see the discussion below under Product Testing).

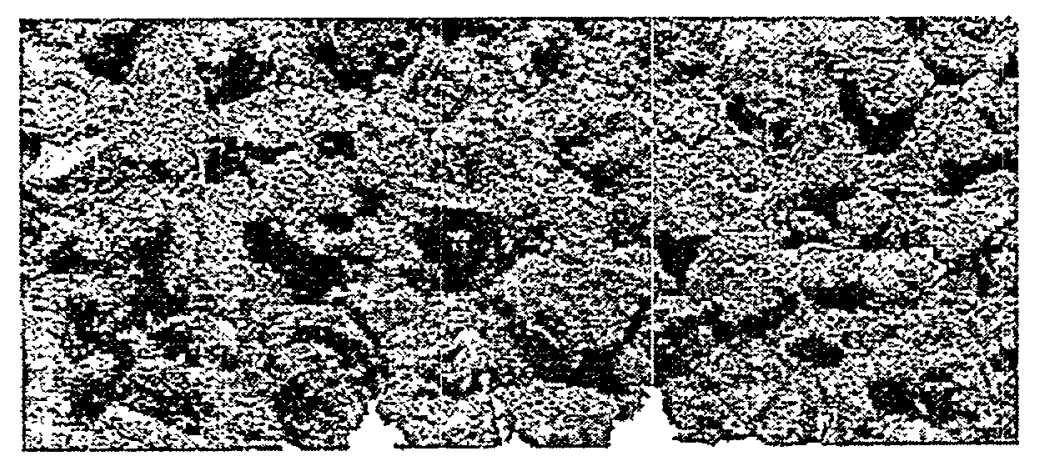

Recovered ASR PUF after Cleaning

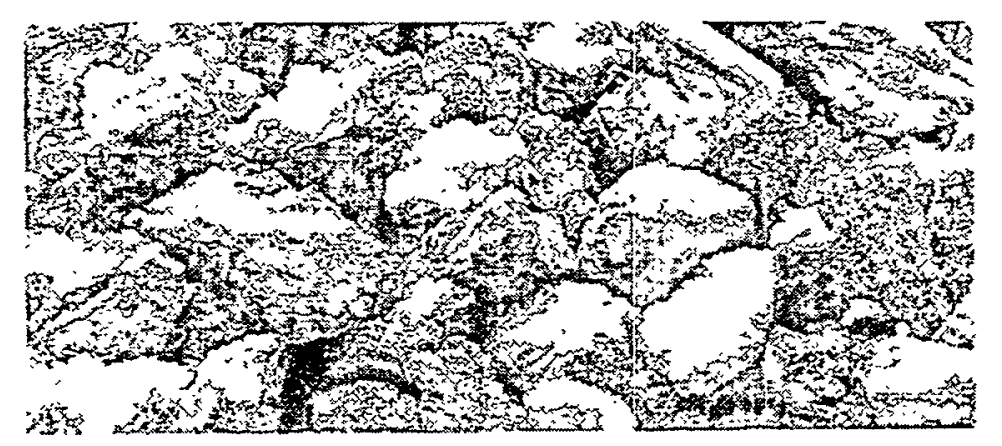

\section{Recovered ASR PUF after Cleaning}

Figure 2 Polyurethane Foam (PUF) as Recovered from Shredder Residue and after Cleaning 


\subsubsection{Batch Process Pilot-Scale Testing}

After the bench-scale work, pilot-scale testing was done to provide rebond market customers with large samples for production testing. The objective of this stage was to confirm that large quantities of foam could be cleaned to meet the requirements of the rebond market and to confirm that the recovered foam could be reused successfully with the existing processes of the foam rebonders.

To produce large quantity samples (i.e., 200-lb bales of recovered and cleaned foam), Argonne built a batch pilot-plant by using modified conventional dry cleaning equipment (washing machine and dryer). The cleaning solution used was perchloroethylene, the conventional dry cleaning solvent. The equipment also included a distillation unit (for regenerating and recovering the perchloroethylene) and a foam baler. The baler densified the foam into $200-300-\mathrm{lb}$ bales for shipment.

The equipment was installed at a shredding facility and operated to produce more than $4000 \mathrm{lb}$ of clean foam for evaluation and testing by foam rebonders. Although rebonders found that the recovered foam product was acceptable (see Section 2.9), operation of this batch pilotplant revealed the following process limitations:

1. Labor requirements for a batch process would be cost-prohibitive in a full-scale operation. A fully continuous process was required.

2. Perchlorethylene was effective as a cleaning agent for the foam, and the solvent could be regenerated and recovered at more than a $99 \%$ recovery rate. However, the dirt removed from the foam during the cleaning process contained trace amounts of perchloroethylene. At a perchloroethylene concentration of 7 or more parts per million, the dirt would have to be disposed of as a hazardous waste. Disposal of the dirt as a hazardous waste would be cost-prohibitive. An alternative cleaning solution was required.

3. Using conventional equipment to dry the foam was time-consuming, requiring residence time of more than $3 \mathrm{~h}$ for effective drying. This drying time would result in cost-prohibitive capital costs for the drying equipment. A new dryer design was required.

\subsubsection{Continuous Process Pilot-Scale Testing}

A continuous wash, rinse, and drying process with a design capacity of $100 \mathrm{lb} / \mathrm{h}$ was designed and tested to confirm that the limitations identified during the batch-process pilot tests were eliminated. The equipment is described in more detail below as part of the overall foamrecovery process that has evolved from this development effort. The wash, rinse, and drying equipment essentially consists of three linear conveyors. In each of the three conveyors, the foam is compressed sequentially and released to mechanically assist in the washing, rinsing, and drying of the foam. The residence time for drying the foam in this equipment is less than $15 \mathrm{~min}$, while in the conventional rotary drum dryer, drying time is $3 \mathrm{~h}$. 
A bench-scale testing campaign was conducted to screen environmentally acceptable aqueous surfactants and detergents that can be used to clean the foam as an alternative to perchloroethylene. Although other organic solvents had been screened during the early benchscale research discussed above, the other organic solvents were cost-prohibitive because of the high cost of explosion-proof equipment that would have been required. Aqueous-based surfactants and detergents that were tested include DowFax, Tauranol ML, Triton SP190, Alcodet 260, Igepal CO-630, a mixture of Alcodet 260 and Igepal CO-630, Biosolve, Triton SP135, Niaproof 08, Aquatek RP-2101, Gemtex SC-77, and Alconox. Most of these cleaning agents were successful in cleaning the foam, given enough time and surfactant. SP190 was able to achieve a high level of cleanliness in a short residence time and at temperature and $\mathrm{pH}$ conditions that do not adversely affect foam quality. The emulsified "oils" were easily separated from the water solution containing this surfactant by de-emulsification at acidic $\mathrm{pH}$. This surfactant was used in the continuous pilot-scale tests.

The continuous $100-\mathrm{lb} / \mathrm{h}$ pilot equipment was installed at a shredder location and operated for about three months. The pilot facility was successfully operated continuously in staged campaigns. At the conclusion of the test, about $10,000 \mathrm{lb}$ of dirty PUF was successfully recovered and cleaned and supplied to the foam rebond market for testing and evaluation. This product also met the quality requirements of the rebond market. A product data sheet was also prepared (see Appendix D).

Basic design considerations of the process are discussed in Appendix E and reference [18]. The resultant overall foam recovery' process (including mass balances, waste streams, and process costs) is discussed in Section 2.3.

\subsection{Polyurethane Foam Recovery Process}

The overall process consists of six basic unit operations: (1) ASR screening and PUF recovery, (2) sizing, (3) washing, (4) rinsing, (5) drying, and (6) baling, as shown in Figure 3. The heart of the system is the patented linear continuous wash, rinse, and dry equipment of unique design, which is described more fully below.

\subsubsection{Unit Operation 1: ASR Screening and PUF Recovery}

PUF can be recovered from ASR in any number of mechanisms, including hand-picking. However, the more cost-effective approach is to recover the PUF from the non-ferrous material stream that is typically conveyed to an eddy-current separator for the recovery of non-ferrous metals in many shredder facilities. In this arrangement, the non-ferrous fraction from the shredder will contain all material, except the ferrous metals recovered magnetically. Typically, this material is fed to a trommel to reject $-5 / 8$-in. fines before the stream reaches the eddy current separator. Following the eddy current separator, the non-metallic fraction or reject from the eddy-current separator would be fed to a slotted trommel that is similar in design to the second stage of the two-stage trommel designed by Argonne (see Appendix A) for 


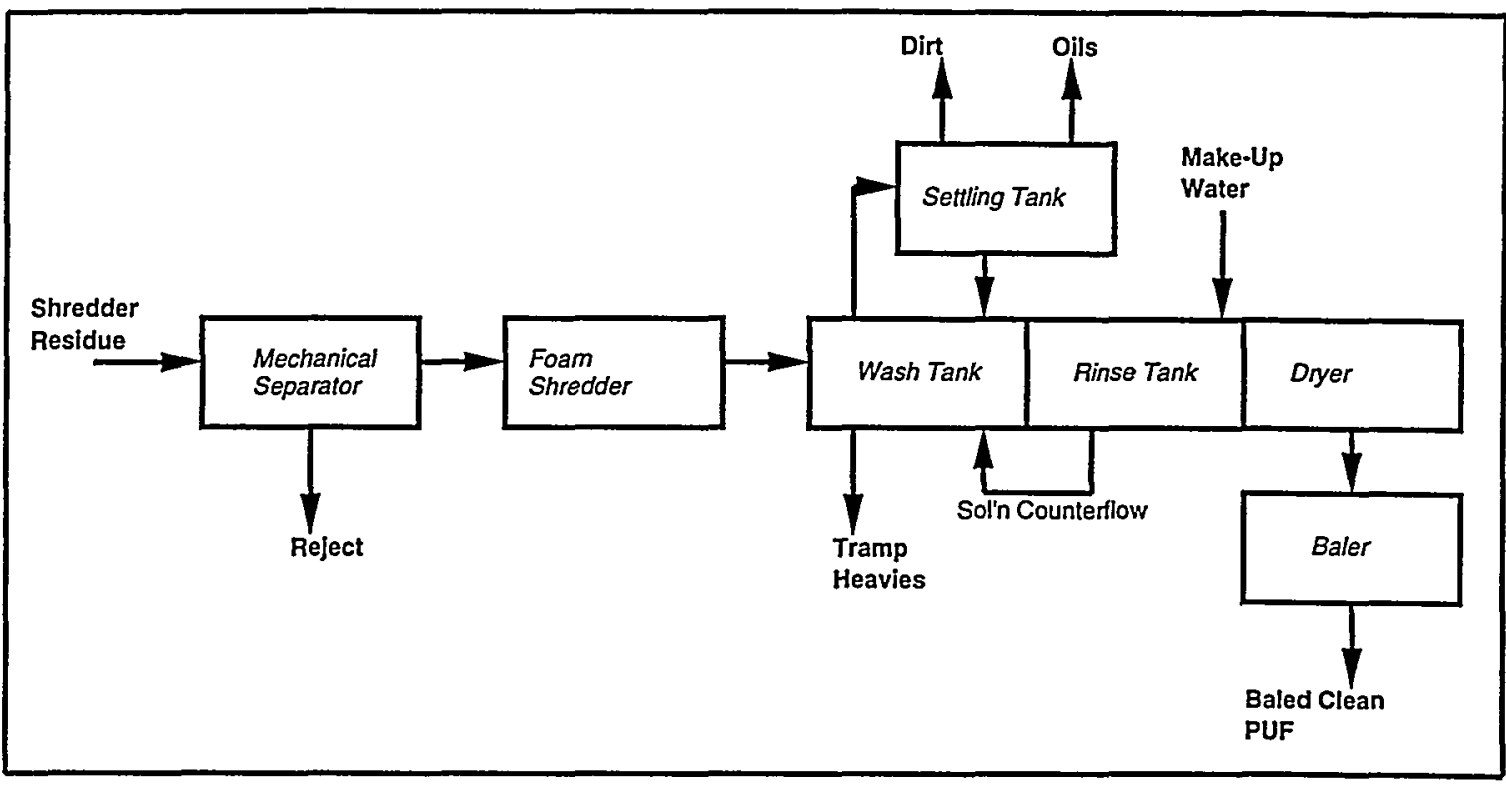

Figure 3 Block Flow Diagram of the PUF Recovery Process

separating dirty PUF from shredder residue. The recovered foam at this stage will vary considerably in physical dimensions.

\subsubsection{Unit Operation 2: PUF Sizing}

The PUF is then conveyed by belt to a shredder to reduce all of the foam to a consistent size (pieces of about 2-5 in.). Size reduction is necessary for effective cleaning and subsequent drying of the foam and helps to liberate entrained dirt and metals. The sized foam is then conveyed to the washing operation.

\subsubsection{Unit Operations 3, 4, and 5: Washing, Rinsing, and Drying}

Washing, rinsing, and drying of the sized foam is accomplished in a continuous, threestage linear system, as shown in Figure 4.

The photograph (Figure 4) shows the equipment used during testing at Argonne (before the field test was conducted in the latter half of 1997). As shown in the photograph, the sized foam is conveyed into a hopper that meters the sized foam into the wash station. The wash station, located between the end of the conveyor and just on the other side of the two personnel on the steps, consists of two sections. In the first section, heavy tramp is removed from the sized foam. The foam floats and moves into the wash section via solution flow. In the wash section, an indexed squeeze conveyor moves the foam forward to the rinse station while compressing and releasing the foam at about 20 cycles/min. This process ensures that any entrained oils and dirt are removed. The design of the rinse station is the same as that of the wash section of the wash tank. In the photograph, clean foam, which is pale, is exiting the rinse 


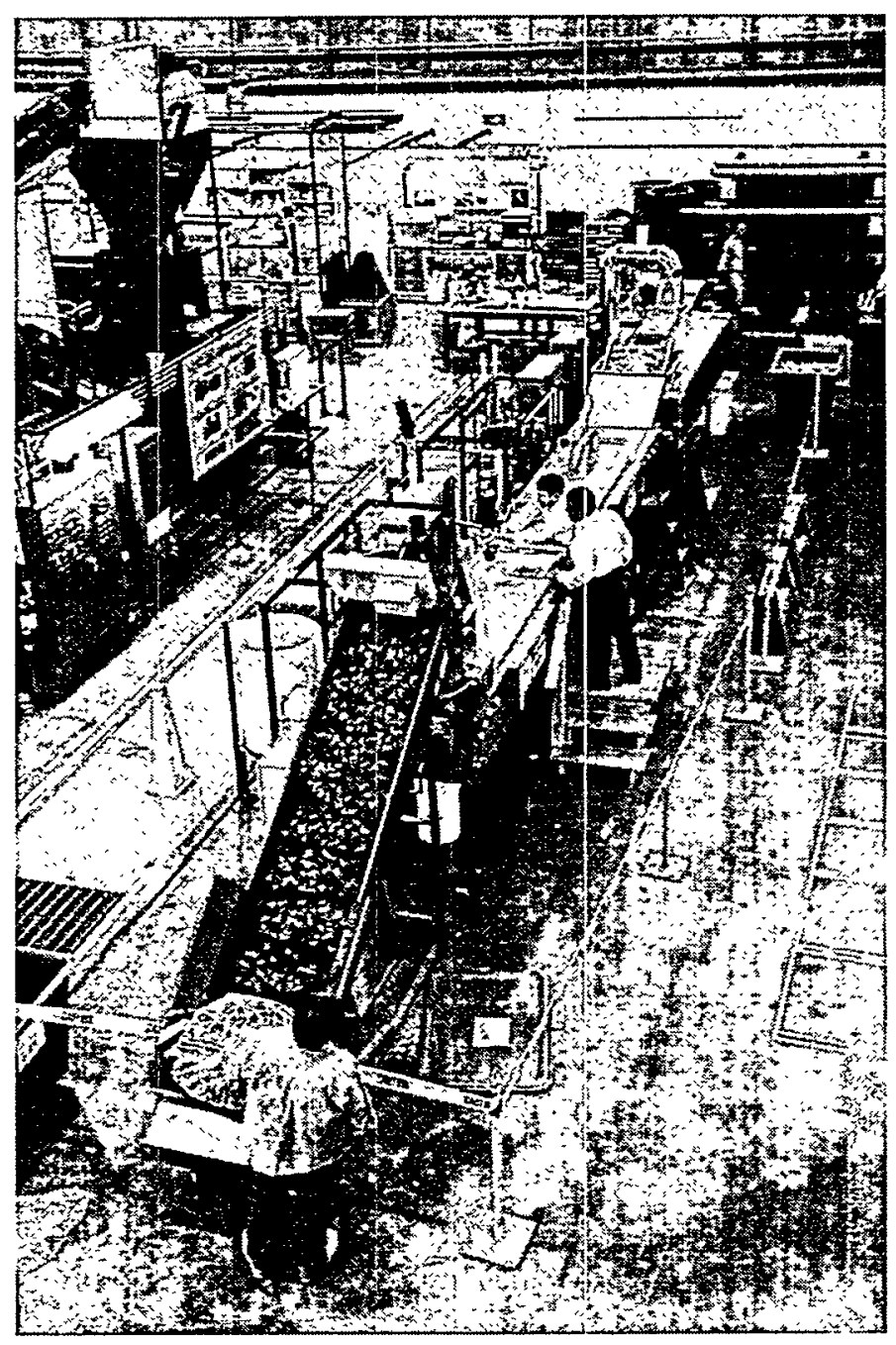

Figure 4 Wash, Rinse, and Dry Continuous Equipment

tank as it is being fed into the linear dryer at the very top right of the photograph. The dryer is also a conveyor system, with a series of rollers above the conveyor that compress and release the foam. This type of action is essential to accomplish drying of the PUF in any reasonable residence time. In a conventional rotary dryer, a residence time of over $3 \mathrm{~h}$ is required. In the linear squeeze dryer designed by Argonne staff, the residence time is less than $15 \mathrm{~min}$.

Washing: The sized foam is then washed in a linear washing machine that automatically conveys the foam through the machine while subjecting the foam to a series of squeezes to reject entrained dirt. The wash tank consists of two sections. In the first section, any tramp metals or other heavies are allowed to settle as the foam is moved to the washing section. Tramp materials are screw-conveyed from the bottom of the tank. The aqueous-based washing solution contains a surfactant for the liberation of entrained oils and dirt. The temperature of the wash water is maintained between $130^{\circ} \mathrm{F}$ and $180^{\circ} \mathrm{F}$, depending upon the surfactant being 
used. The dirty wash water cycles through hydrocylones and to a settling tank, in which water is recycled continuously back to the wash tank. Oils are skimmed and entrained solids are screw-conveyed from the bottom of the settling tank for disposal. Typically, producing 500 tons of clean foam will require processing about 600 tons of dirty foam. The dirty foam will contain about 75 tons of entrained wet dirt and about 25 tons of entrained oils.

Rinsing: The rinsing operation is essentially the same as the washing operation. Rinsing is done with only $180^{\circ} \mathrm{F}$ water. Foam from the rinse tank is discharged to the dryer and passes through a pair of soft rubber-covered squeeze rolls. A vacuum is also induced on the foam as it is squeezed to prevent the foam from re-absorbing the water. This de-watering mechanism reduces the water carryover from the rinse tank down to about $0.6 \mathrm{lb}$ per pound of foam.

Drying: The clean foam is then dried in a linear squeeze conveyor dryer. Residence time in the dryer is less than $15 \mathrm{~min}$; within this period, the retained moisture content is less than two weight per cent in the foam. Heated air is used to dry the foam.

\subsubsection{Unit Operation 6: Baling}

The clean, dry foam is then conveyed to a horizontal baler to densify the foam for shipment to the customer. A horizontal continuous baler is used to produce bales that weigh between 200 and $300 \mathrm{lb}$ with dimensions of $2 \mathrm{ft}$ by $2 \mathrm{ft}$ by $5 \mathrm{ft}$ (Figure 5). Foam balers that can produce $800-1 b$ bales are also commercially available.

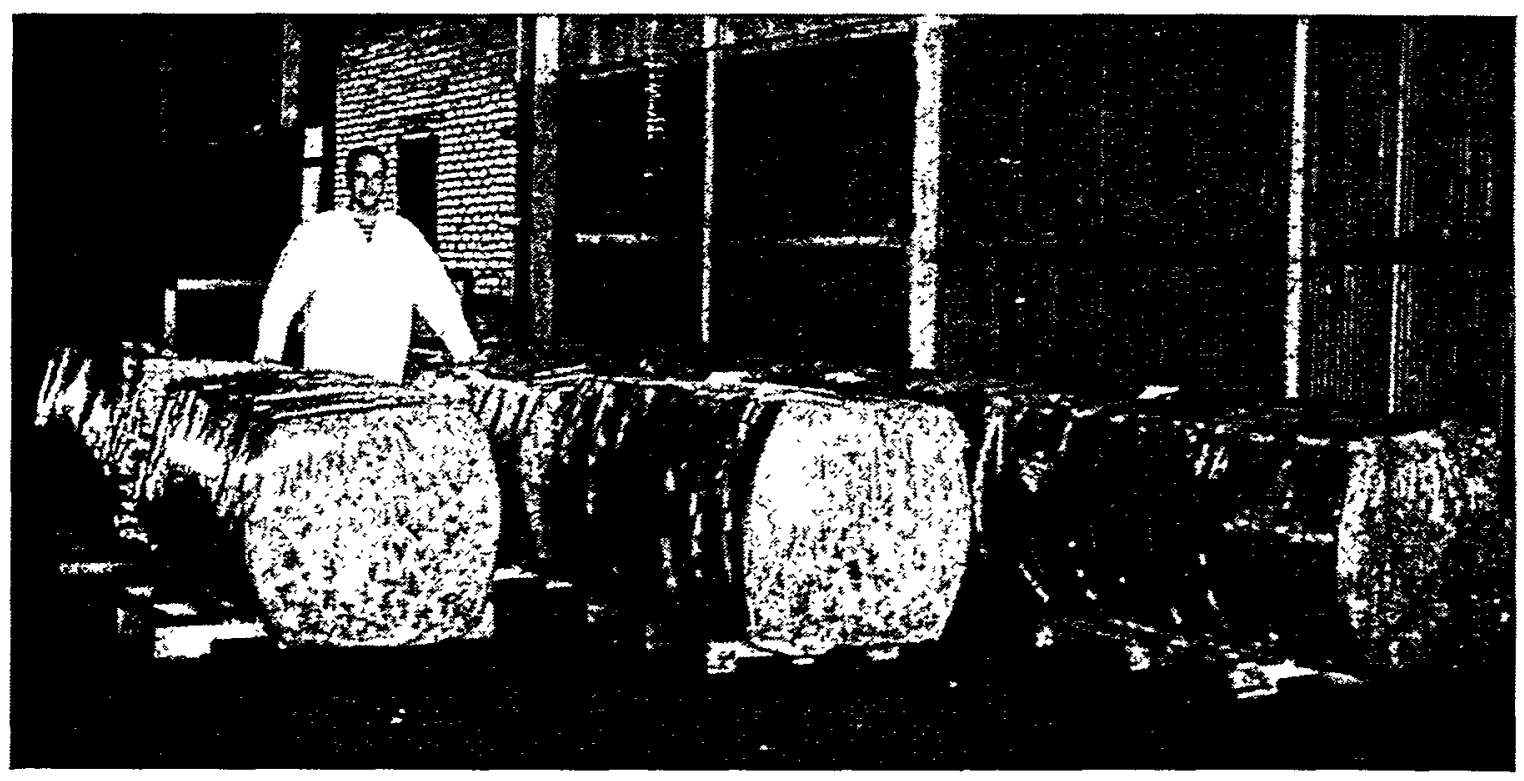

Figure 5 Clean PUF Bales 


\subsection{Process Mass Balance}

Producing $100 \mathrm{lb}$ of clean and dry PUF typically requires processing about 3,000 lb of ASR. The actual yield of clean PUF will, of course, vary at each shredder, depending on the mix of obsolete cars relative to other materials being shredded and the type of shredding process. Typically, 3,000 lb of ASR will yield about $150 \mathrm{lb}$ of PUF material. The balance of the stream contains plastics, fines, and other materials. The PUF material will be fed to a foam shredder for size reduction before it enters the wash tank of the continuous foam-washing and drying process. The PUF material will contain about $127 \mathrm{lb}$ of dirty foam and about $23 \mathrm{lb}$ of tramp material (such as wire, metal, plastics, and rubber).

Cleaning the dirty foam will require about $1 \mathrm{lb}$ of chemicals (surfactants, detergents, etc.) and about $115 \mathrm{lb}$ (14 gal) of make-up water. About $78 \mathrm{lb}$ (or about $9.5 \mathrm{gal}$ ) of the make-up water is required to offset the evaporative water losses, and the balance of the make-up water is dragged out with the reject streams. In the process, the following reject streams are generated (Figure 6):

1. Heavy solids from the Wash Tank (23lb). These solids are pieces of rubber, plastics, and metals that are carried over with the PUF during the separation of the PUF from the ASR. These solids contain about $20 \mathrm{wt} \%$ non-ferrous metals. These materials will be allowed to settle to the bottom of the wash tank, and then they will be screw-conveyed from the wash tank for further processing to recover the metals and maybe some of the plastics.

2. Dirt and oils entrained in the PUF (40 lb). The solid dirt will be recovered from the wash water and rinse water settling tanks. Oils will be skimmed from the wash water settling tank or recovered from an ultra-filtration unit treating the water in this settling tank. The mass of the dry solid waste is about $21 \mathrm{lb}(35 \mathrm{lb}$ wet). The oils stream (about $5 \mathrm{lb}$ ) contains about $1 \mathrm{lb}$ total solids, more than $2 \mathrm{lb}$ water, and less than $1 \mathrm{lb}$ automotive fluids.

3. Waste water $(25 \mathrm{lb}$ ). About $25 \mathrm{lb}$ of waste water will be extracted from the wash tank and replaced with water from the rinse tank. The same amount of fresh water will be added to the rinse tank to maintain clean wash and rinse water.

4. Water loss by evaporation $(78 \mathrm{lb})$. Water loss by evaporation is primarily from the dryer $(61 \mathrm{lb})$. In addition, about $17 \mathrm{lb}$ of water is evaporated from the rinse and wash tanks.

\subsection{Disposition of the Process Reject/Waste Streams}

1. Heavy solids from the Wash Tank (23 lb). This stream is not considered a waste stream from the foam cleaning process. It consists essentially of plastics and metals carried over from the ASR. Therefore, it will be returned to the shredder for further processing to recover the metals and maybe the plastics. 


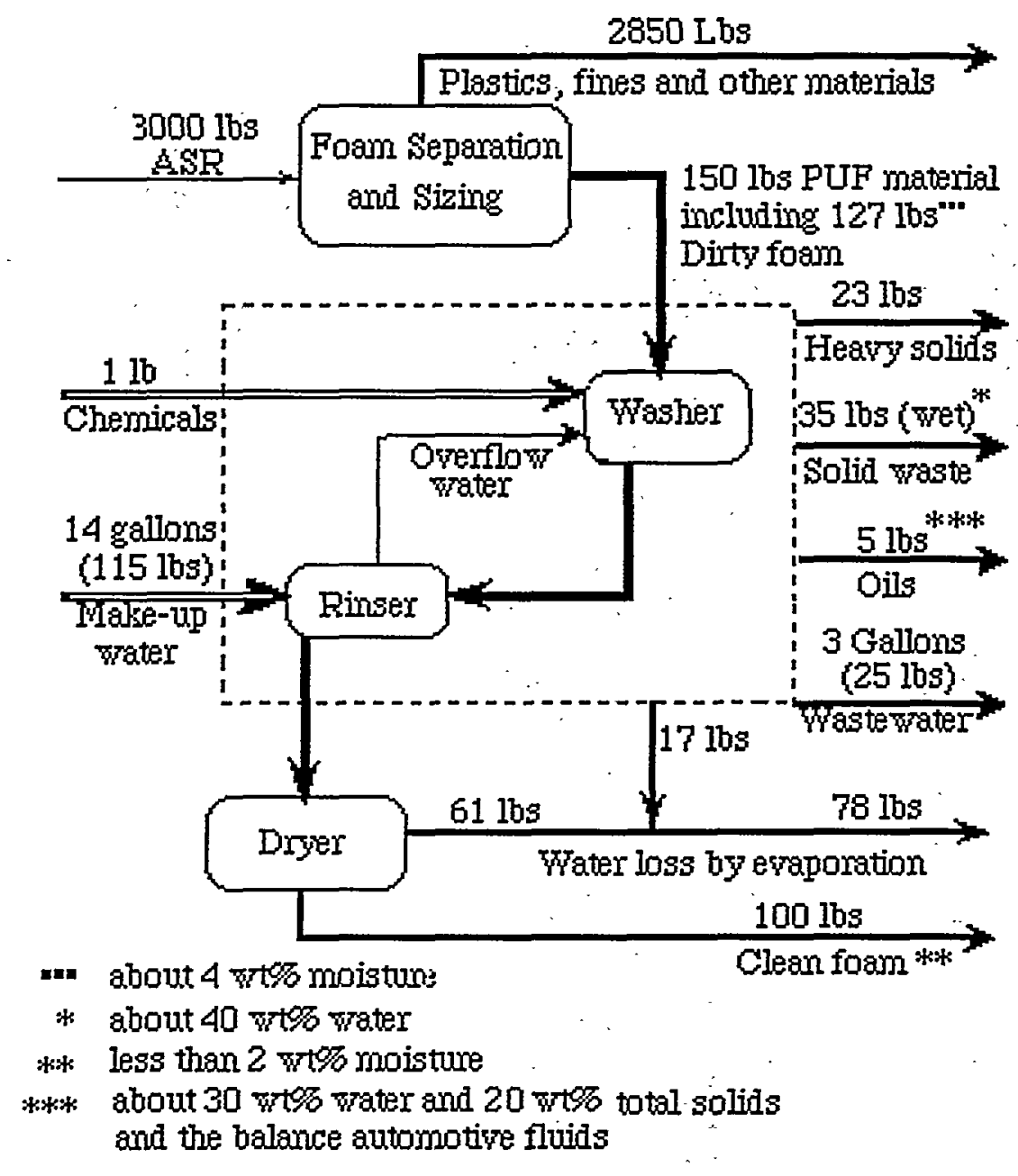

Figure 6 Process Flow Sheet with Material Balance (basis: $100 \mathrm{lb} / \mathrm{h}$ of clean foam)

2. Solid Waste. The amount of solid waste (dirt) from the wash water and rinse water setting tanks is about $21 \mathrm{lb}$ ( $35 \mathrm{lb}$ wet). This stream is rich in iron-oxide and silicon-oxide, and it is made mostly of fine particles (less than 0.25 in.). Because these solids are washed as the foam is washed, they contain fewer oils and other organics, such as PCBs, than the original ASR (this was confirmed by TCLP analysis). Because the material passed TCLP testing, it could be used as a source of iron-oxide units, or it could be disposed of in landfills, along with regular ASR. (The TCLP test results are given in Table F.1 in Appendix F.)

3. Oils. The amount of oils, or oily water, skimmed from the wash water settling tank is about $5 \mathrm{lb}$, of which about $2 \mathrm{lb}$ is water. In the field test, the oily water was separated by using an ultra-filtration unit. TCLP analysis on this stream was conducted, and it passed TCLP testing. (The results are given in Table F.2 in Appendix F.) 
4. Waste water. This stream consists of about $25 \mathrm{lb}$ of waste water extracted from the wash tank. TCLP analysis on this stream was also conducted, and it passed TCLP testing. (The results are given in Table F.3 in Appendix F.) This stream contains about 3.7 wt\% total solids and about $42 \mathrm{mg} / \mathrm{L}$ total zinc. This material is also not hazardous and can be disposed of as sewer water, unless the specific requirements of the local municipality do not allow it.

\subsection{Process Costs}

Process economics are estimated on a design basis of 1,000 ton/yr of clean foam produced in an eight-hour shift operating 300 days/yr. This amount is equivalent to a design capacity of about $840 \mathrm{lb} / \mathrm{h}$, which equates to the estimated amount of flexible foam that can be recovered from about 60 shredded cars per hour.

The total capital investment is estimated at $\$ 700,000$ for all unit-operations equipment. This investment includes the cost of the PUF trommel, the PUF shredder, the continuous washing and drying equipment, and the foam baler. The cost does not include contractor's engineering fees, license fees, or taxes and permit fees.

Approximately two barrels of oil waste water would be produced each day and would have to be disposed of by a waster handler. The cost is estimated at $\$ 100 / \mathrm{bbl}$, on the basis of pilotplant data.

Table 2 PUF Recovery Process Costs

\begin{tabular}{|lr|}
\hline \multicolumn{1}{|c|}{ Type of Expenditure or Earning } & $\begin{array}{c}\text { Expenditure or } \\
\text { Earning (\$) }\end{array}$ \\
\hline Annual Revenues @ \$0.30/b foam & 600,000 \\
Credit for Avoided Disposal @ \$20.00/ton & 20,000 \\
Total Annual Revenues & 620,000 \\
& \\
Operating Costs: & \\
Labor, 2 operators at \$18/h & 86,400 \\
Electricity, 150 kWh/h @ $\$ 0.10 / \mathrm{kWh}$ & 36,000 \\
Natural Gas, 1 million Btu/h @ \$4.00/million Btu & 9,600 \\
Chemicals, 0.01 Ib/b foam @ \$0.85/bb & 17,000 \\
Disposal Costs for Wet Dirt @ \$20.00/ton & 8,000 \\
Disposal Costs for Oils @ \$100.00/barrel & 63,500 \\
Maintenance, @ 3.5\% of Capital & 22,750 \\
Total Annual Operating Costs & 243,250 \\
& \\
Annual Earnings before Interest, Taxes, & 376,750 \\
Depreciation and Amortization & \\
\hline
\end{tabular}




\subsection{Market Potential for Recycled Foam}

In North America, about 2,100 million pounds of PUF is used per year [17]. The demand for foam in North America has been increasing because of increasing domestic production of vehicles (Figure 7). The largest customer for foam products is the automotive industry, which uses about 500 million $\mathrm{lb} / \mathrm{yr}$ for various applications, including carpet padding, seat cushions, sound insulation, and interior dash and trim panel padding.

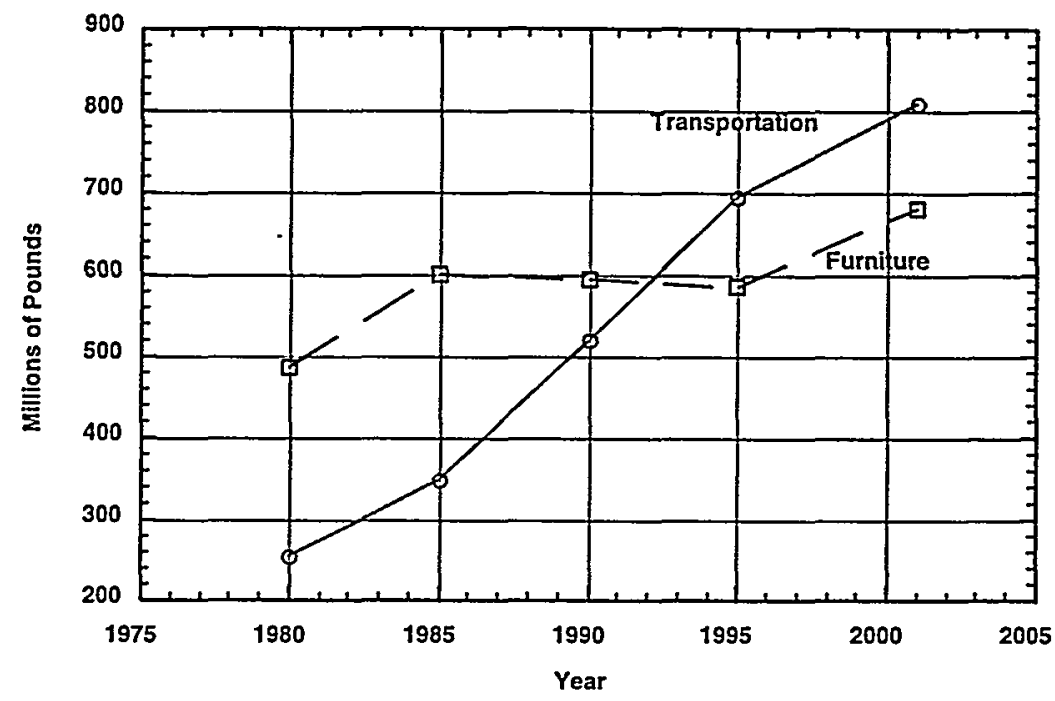

Figure 7 U.S. Foam Consumption by the Transportation and Furniture Industries

The supply of foam is typically met from about 1,750 million $1 \mathrm{~b} / \mathrm{yr}$ of "virgin" foam produced from chemicals, 200 million $\mathrm{lb} / \mathrm{yr}$ of prompt scrap foam from the domestic market, and 150 million lb/yr of prompt scrap foam imported from Europe and Asia. The estimated cost of virgin foam is about $\$ 0.80-1.50 / \mathrm{lb}$. Scrap foam prices are quite volatile and can vary from $\$ 0.15$ to $\$ 0.60 / \mathrm{lb}$. On average, the long-term price for scrap foam is estimated at $\$ 0.30 / \mathrm{lb}$.

\subsection{Market Opportunity/Supply Side}

There are an estimated 185 shredding operations located throughout the United States. The total amount of foam potentially recoverable from these operations is estimated at $300-400$ million $\mathrm{lb} / \mathrm{yr}$.

For the process to be viable, however, the minimum annual quantity of foam recovered from a single site must exceed 1.5 million $\mathrm{lb}$. About $45 \%$ of the shredders in the United States are not expected to meet this threshold production potential. The distribution of the number of shredders and the total percent of foam recoverable as a function of the annual PUF recovery potential per shredder is shown in Figure 8. For example, 9\% of all shredders are expected to process only enough vehicles that would yield less than 1 million $\mathrm{lb} / \mathrm{yr}$ of foam. Only $5 \%$ of the PUF recoverable from all shredders is recoverable from the smallest $9 \%$ of the shredders. 
About $45 \%$ of all shredders do not have the potential to produce at least 1.5 million lb/yr of PUF. The amount of PUF potentially recoverable from this $45 \%$ is approximately only $30 \%$ of the PUF recoverable for all shredders.

The market potential for the foam recovery process will also be constrained by the type of shredding operation and, in particular, to the extent that the shredder uses water for dust control during the shredding operation. On the basis of our experience, we estimate that $25 \%$ of the shredding operations are such that the foam recovery process would not be cost-effective. If this is the case, then the upper limit on the market potential for the Argonne foam recovery process is about $50 \%$ ( $70 \%$ potential after production threshold cut of $75 \%$ because of operations*) of the total foam potentially recoverable from U.S. shredders.

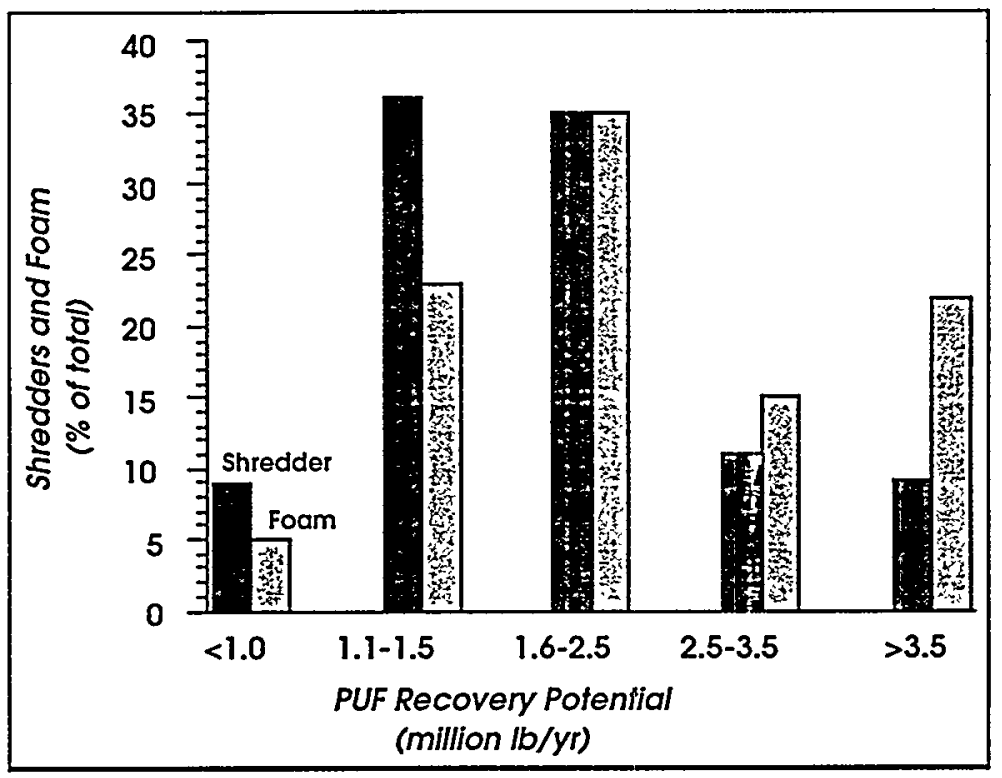

Figure 8 Distribution of Shredders and Foam Recovery Potential (as a per cent of total) as a Function of the Annual Recovery Potential per Plant Site

\subsection{Recycled Foam Product Testing}

During each stage of the process development effort, samples of the recovered and processed foam were supplied to potential customers to confirm the acceptability of the foam for reuse.

* This would indicate that the market potential for the Argonne foam recovery process is about 150-200 million pounds per year. 
At the initial stages, small samples (less than a few pounds) were supplied for evaluation. At that stage, the principal objective was to visually inspect the sample to determine if the foam was sufficiently clean. The foam had to meet three criteria: (1) visual cleanliness with no entrained metals, (2) no odor, and (3) no entrained oils. In terms of these three criteria, foam produced by the bench-scale work was equal to or better than prompt scrap foam from other sources.'

Larger quantities of foam were supplied subsequently for more quantitative testing. Samples of the material produced by the batch pilot plant were tested for strength properties, odor, and fogging. The results were all in the acceptable range for many automotive applications. The next step was to make vehicle components with the recycled foam for testing as sound-suppression products. These products were tested in three 1997 models (Jeep Cherokee, Mercury Villager, and Saturn). The performance of the recycled foam was compared with that of fiberpad material. The results are shown in Figure 9. The acoustic performance of the recycled foam ( $6 \mathrm{lb} / \mathrm{ft}^{3}$ Argonne foam) was equivalent to that of the conventional fiberpad.

Large samples of foam were submitted to several scrap-foam rebonders during the project for evaluation (Figure 10). Cleaned foam was sent to a foam rebonder that processed it into foam logs weighing about $750 \mathrm{lb}$ each; the rebonder processed the foam in exactly the same manner as it would process prompt foam scrap, using the same ratio of steam and chemicals. The foam logs were then allowed to dry, and then they were sliced into different densities and

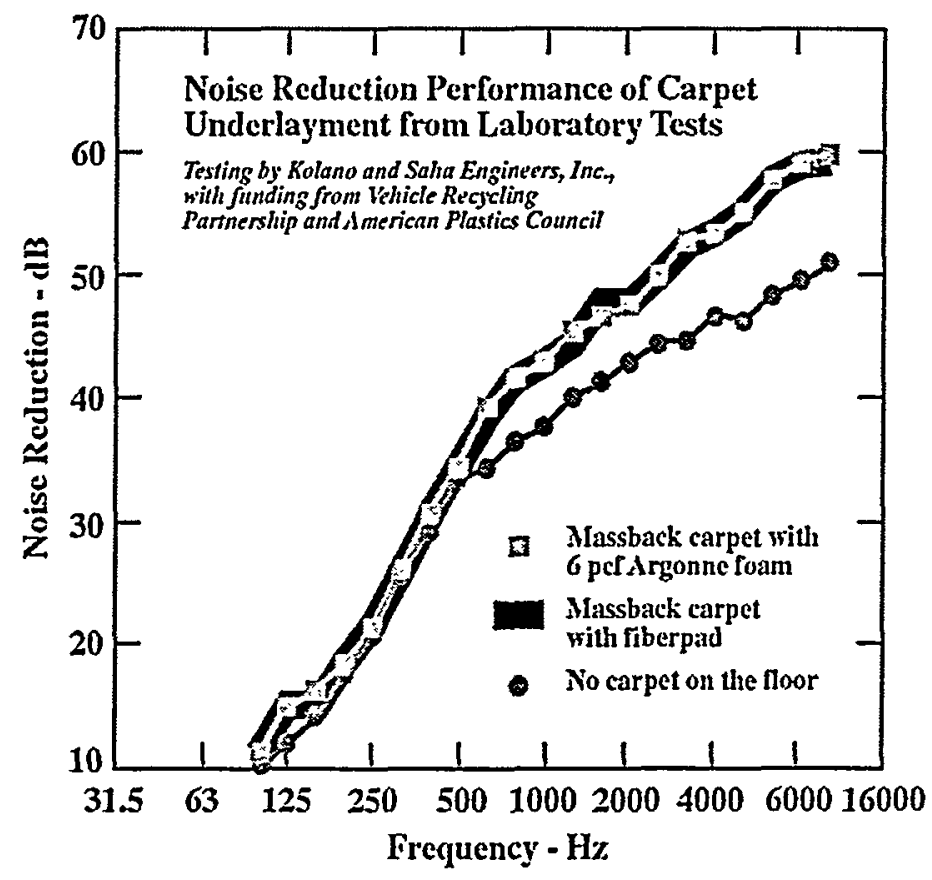

Figure 9 Noise Reduction of Automotive Carpet Underlayment 

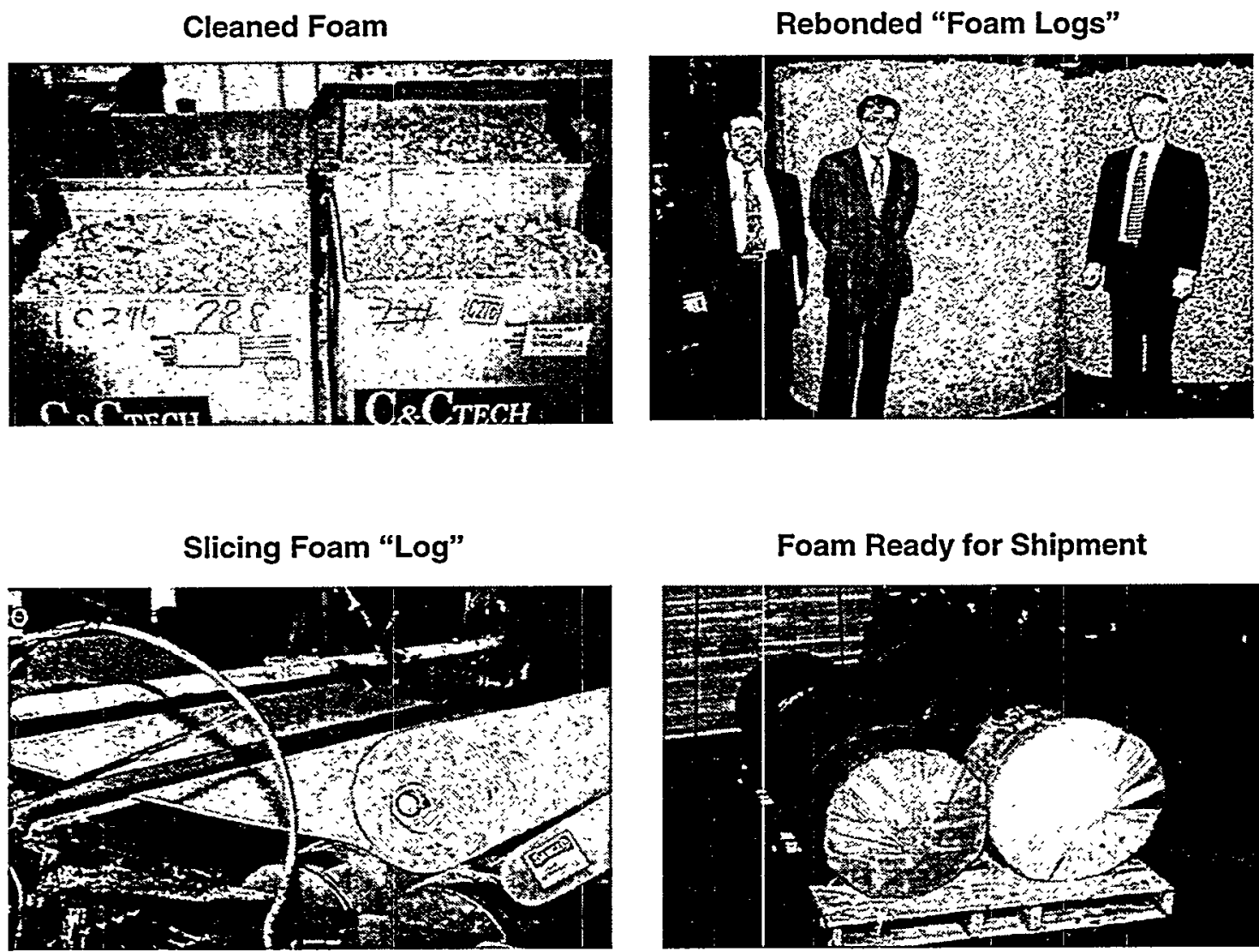

Foam Ready for Shipment

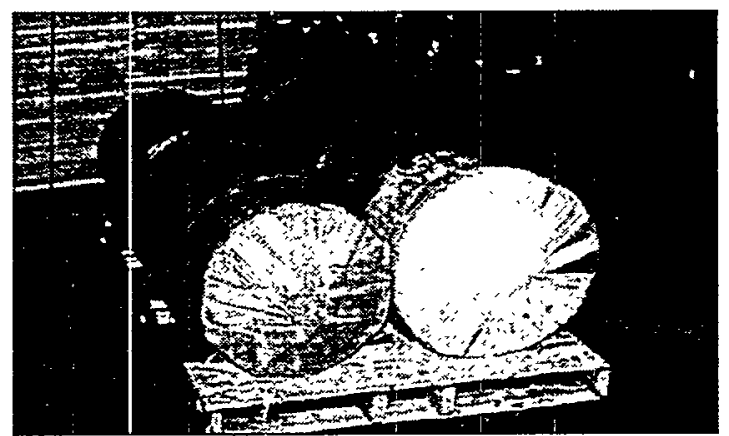

Figure 10 Recovered Foam from ASR Made into Carpet Padding

prepared for shipping. Some of the products made from these samples are being tested in a number of vehicles in the United States and Canada [19]. 


\section{Section 3 \\ Conclusions}

At Argonne, staff have conceived of, developed, and tested a process for recovering PUF from shredder residue. The process has evolved from bench-scale proof-of-concept to successful process demonstration at a shredding facility. During process development, samples of recovered PUF were supplied to potential customers. Potential customers found that the PUF recovered by means of the Argonne process met their quality requirements and performed as well as competing virgin materials and prompt industrial scrap foam.

Argonne's cost analysis, which is based on pilot-plant operations using vendor quotes, indicates the capital cost for a 1,000 -ton-per-year PUF plant is about $\$ 700,000$. The payback period on this investment is estimated to be less than two years, on the basis of a PUF market price of $\$ 0.30 / \mathrm{lb}$. 


\section{Section 4 References}

1. Jody, B.J., E.J. Daniels, and A.P.S. Teotia, 1996, "Progress in Recycling of Automobile Shredder Residue," Chapter 5, Conversion and Utilization of Waste Materials, M. Rashid Khan (editor), Taylor and Francis, Washington, D.C., May (invited).

2. Jody, B.J., et al., 1994, "Recovering Recyclable Materials from Shredder Residue," Journal of Metals 46(2):40-43, Feb.

3. Jody, B.J., E.J. Daniels, and J.A. Pomykala, Jr., 1996, Progress in Recycling of Automobile Shredder Residue, Proc. Extraction and Processing Division, TMS Annual Meeting, Anaheim, Calif., Feb.

4. Jody, B.J., E.J. Daniels, and N.F. Brockmeier, 1994, "Recovering Plastics from Cars," ChemTech 24(11):41-44, Nov.

5. Daniels, E.J., 1994, "Separation and Recovery Process R\&D to Enhance Automotive Materials Recycling," in Proc. 3rd International Conference on Automobile Recycling, Auto Recycle Europe '94, Milano, Italy, May 5-6.

6. Jody, B.J., et al., 1994, "Recovering Recyclable Materials from Shredder Residue," in Proc. 1994 Minerals, Metals, and Materials Conference, Extraction and Processing Division, San Francisco, Calif., Feb. 17-March 3.

7. Daniels, E.J., B.J. Jody, and P.V. Bonsignore, 1992, "Alternatives for Recycling Automobile Shredder Residue," The Journal of Resource Management and Technology 20(1):14-26, March.

8. Jody, B.J., E.J. Daniels, and P.V. Bonsignore, 1992, Treatment and Recycling of Shredder Fluff: Final Report on Phase I, Proof of Concept, ANL/ESD-13, Argonne National Laboratory, Argonne, Ill., Feb.

9. Jody, B.J., and E.J. Daniels, 1991, “Automobile Shredder Residue: Treatment Options," Hazardous Waste \& Hazardous Materials 8(3):219-230, Sept.

10. Jody, B.J., E.J. Daniels, and P.V. Bonsignore, 1991, "A Chemical Approach to Recycling Mixed Plastics from Shredder Fluff," in Proc. 6th Annual Recycling Plastics Conf., Plastics Institute of America, Washington, D.C., May.

11. Bonsignore, P.V., B.J. Jody, and E.J. Daniels, 1991, "Separation Techniques for Auto Shredder Residue," in 1991 SAE International Congress and Exposition, SAE Technical Paper No. 910854, pp. 59-63, Feb. (invited). 
12. Daniels, E.J., et al., 1990, "Auto Shredder Residue: Process Development for Recovery of Recyclable Constituents," in Proc. Sixth International Conference on Solid Waste Management and Secondary Metals, Philadelphia, Pa., Dec.

13. Jody, B.J., and E.J. Daniels, 1990, "Recycling of Plastics in Automobile Shredder Residue," in Proc. 25th Intersociety Energy Conversion Engineering Conf., Reno, Nev., Aug.

14. Arzoumanidis, G.G., et al., 1995, "Catalytic Pyrolysis of Automobile Shredder Residue," presented at 210th National Meeting of the American Chemical Society, Chicago, Ill., Aug. 20-25.

15. Brockmeier, F.E., and M.J. McIntosh, 1994, "Economic Evaluation of Auto Shredder Residue Pyrolysis Plant Design Using Process Simulation Software," presented at American Institute of Chemical Engineers Annual Meeting, San Francisco, Calif., Nov. 1318.

16. Jones, F.L., 1994, "Pyrolysis of Automobile Shredder Residue," Cogen Designs, Inc., Nov.

17. "Plastics Recycling," Chemical Economics Handbook, 1997, SRI Consulting, Menlo Park, Calif., May.

18. Jody, B.J., E.J. Daniels, and J. Libera, 1999, "Efficient Continuous Dryer for Flexible Polyurethane Foam and Cleaning Apparatus," U.S. Patent No. 5,882,432, March 16.

19. Blair, G.R., 1998, "Recovery and Re-use of Automotive Shredder Residue Flexible Polyurethane Foam," Woodbridge Foam Corp., Ontario, Canada. 


\section{Section 5 \\ Annotated Bibliography of Argonne Shredder Residue Research}

Jody, B.J., E.J. Daniels, and A.P.S. Teotia, 1996, "Progress in Recycling of Automobile Shredder Residue," Chapter 5, Conversion and Utilization of Waste Materials, M. Rashid Khan (editor), Taylor and Francis, Washington, D.C., May (invited).

The authors summarize the literature on alternatives for recycling shredder residue. They discuss primary and secondary recycling research, outline various processes (e.g., pyrolysis, hydrolysis, gasification) that have been proposed or investigated for tertiary recycling, and discuss quaternary (waste to energy) recycling research. The authors also discuss their research on separation and solvent extraction for recovery of polymeric materials from shredder residue.

Jody, B.J., E.J. Daniels and J.A. Pomykala, Jr., 1996, Progress in Recycling of Automobile Shredder Residue, Proc. Extraction and Processing Division, TMS Annual Meeting, Anaheim, Calif., Feb.

The authors discuss the status of their research on recovery of polyurethane foam, thermoplastics, and iron-oxides from shredder residue. Physical properties of an ABS recyclate, recovered by solvent extraction, are compared with the physical properties of a virgin-grade $A B S$.

Arzoumanidis, G.G., et al., 1995, "Catalytic Pyrolysis of Automobile Shredder Residue," presented at 210th National Meeting of the American Chemical Society, Chicago, Ill., Aug. 20-25.

Arzoumanidis et al. discuss laboratory research to investigate the effect of different catalysts on the relative distribution of pyro-products and on the composition of the gaseous product. The authors hypothesize that pyrolysis occurs under two distinct regimes: a low-temperature stage at about $300^{\circ} \mathrm{C}$ and a high-temperature stage at about $700^{\circ} \mathrm{C}$.

Brockmeier, F.E., and M.J. McIntosh, 1994, "Economic Evaluation of Auto Shredder Residue Pyrolysis Plant Design Using Process Simulation Software," presented at American Institute of Chemical Engineers Annual Meeting, San Francisco, Calif., Nov. 13-18.

Brockmeier and McIntosh present an engineering cost analysis of six different pyrolysis reactor configurations for conversion of shredder residue to fuels relative to a novel reactor design referred to as a Nonseparatory Double Rotary Kiln reactor. The sensitivities of process costs to such parameters as disposal cost credit, process yield, and product values are presented. 
Jody, B.J., E.J. Daniels, and N.F. Brockmeier, 1994, “Recovering Plastics from Cars," ChemTech 24(11):41-44, Nov.

The authors discuss two approaches to recovering thermoplastics via solvent extraction. IR spectra of recovered ABS, PVC, and a polyolefin mix are compared with standards.

Daniels, E.J., 1994, "Separation and Recovery Process R\&D to Enhance Automotive Materials Recycling," in Proc. 3rd International Conference on Automobile Recycling, Auto Recycle Europe '94, Milano, Italy, May 5-6.

Daniels discusses changes in automotive materials use to reduce weight and increase fuel economy and the resultant impact that those changes can have on the existing recycling infrastructure. Three cases are presented: (1) coated sheet-steels, (2) modified aluminum alloys, and (3) plastics. Research to provide for the recycling of these materials is discussed.

Jody, B.J., et al., 1994, "Recovering Recyclable Materials from Shredder Residue," in Proc. 1994 Minerals, Metals, and Materials Conference, Extraction and Processing Division, San Francisco, Calif., Feb. 17-Mar. 3.

Jody, B.J., et al., 1993, "A Process to Recover Plastics from Obsolete Automobiles by Using Solvents at Ambient Pressure," American Chemical Society.

Jody, B.J., et al., 1992, "Chemical and Mechanical Recycling of Shredder Fluff," in Proc. 1992 Pollution Prevention Conference for Iron \& Steel Industry, Chicago, Ill., Oct. 14-15.

Daniels, E.J., B.J. Jody, and P.V. Bonsignore, 1992, "Alternatives for Recycling Automobile Shredder Residue," The Journal of Resource Management and Technology 20(1):14-26, March. In the above four references, the authors discuss research on solvent extraction for recovery of thermoplastics from shredder residue. The compositions of fines and extracted oils are presented.

Daniels, E.J., B.J. Jody, and P.V. Bonsignore, 1992, "Alternatives for Recycling Automobile Shredder Residue," The Journal of Resource Management and Technology 20(1):14-26, March. The authors conduct a technical and economic comparison of a number of alternatives, including landfill, incineration, materials recovery, and materials conversion via pyrolysis and gasification. They also present a process concept for the recovery of $90 \%$ of ASR materials for recycling.

Jody, B.J., E.J. Daniels, and P.V. Bonsignore, 1992, Treatment and Recycling of Shredder Fluff: Final Report on Phase I, Proof of Concept, ANL/ESD-13, Argonne National Laboratory, Argonne, Ill., Feb.

This report details the initial research of the authors to separate and recover certain materials from shredder residue for recycling. The authors present a basic process concept 
for recovery of certain materials and discuss experimental procedures, apparatus design, and experimental results.

Jody, B.J. and E.J. Daniels, 1991, "Automobile Shredder Residue: Treatment Options," Hazardous Waste \& Hazardous Materials 8(3):219-230, Sept.

The authors review the literature discussing alternative approaches to handling auto shredder residue.

Jody, B.J., E.J. Daniels, and P.V. Bonsignore, 1991, "A Chemical Approach to Recycling Mixed Plastics from Shredder Fluff," in Proc. 6th Annual Recycling Plastics Conf., Plastics Institute of America, Washington, D.C., May.

Bonsignore, P.V., B.J. Jody, and E.J. Daniels, 1991, "Separation Techniques for Auto Shredder Residue," in 1991 SAE International Congress and Exposition, SAE Technical Paper No. 910854, pp. 59-63, Feb. (invited).

Daniels, E.J., et al., 1990, “Auto Shredder Residue: Process Development for Recovery of Recyclable Constituents," in Proc. Sixth International Conference on Solid Waste Management and Secondary Metals, Philadelphia, Pa., Dec.

In the above three references, the authors discuss the structure of the auto shredder industry, summarize prior research relating to handling of shredder residue, and discuss their research on physical separation and solvent extraction of shredder residue. Quantities and compositions of shredder residue are estimated.

Jody, B.J., and E.J. Daniels, 1990, "Recycling of Plastics in Automobile Shredder Residue," in Proc. 25th Intersociety Energy Conversion Engineering Conf., Reno, Nev., Aug.

Proof-of-concept research to recover selected thermoplastics from shredder residue via solvent extraction is presented. 


\section{Appendix A \\ The Argonne Bulk Mechanical Separation Pilot Plant}

To study the behavior of ASR in a mechanical separation system, a batch laboratory classification column with air elutriation was built. This system was used to identify mechanisms that can be implemented to concentrate various fractions of ASR, such as foam, iron-oxide fines, and plastics. Data obtained from this apparatus were used to design and build a 2-ton/h pilot facility. This facility is shown in Figure A.1. The experimental facility has a specially designed multistage trommel, a shredder, barrel/gaylord loaders, and conveyors with magnetic pulleys and a trommel. It also has (not shown in Figure A.1) an eddy current separator and a heavy-duty granulator. The facility offers the flexibility of operating all of the units simultaneously or operating some while the others are de-energized.

The facility can be and has been utilized to size reduce and/or separate a number of materials of interest, including ASR, complete automobile instrument panels, computer housings, radios, and other materials. In the case of ASR, the ASR bypasses the shredder and enters the trommel for separation. The first stage in the trommel is equipped with a 1/4-in. screen to separate the fines that are smaller than $1 / 4$ in. This screen is exchangeable with screens of other sizes when required. The second stage is equipped with longitudinal slots in a staggered arrangement. Flat plastic pieces that are smaller than the width and length of the slots fall through the slots and are conveyed over a magnetic pulley to catch any ferrous pieces that were separated with the plastics. The width of these slots is adjustable by a mobile sleeve. Materials that exit the trommel are passed over a gate that can be adjusted to drop odd-shaped pieces that are smaller than a certain size through the preset gate, while the foam pieces are assisted by an air jet, which causes the pieces to "jump" over the gate. The separated foam is also passed over a magnetic pulley to separate any ferrous contaminants. The three fractions leaving the trommel (fines, plastics-rich, and the foam) can be cleaned and purified to produce recyclable products. 


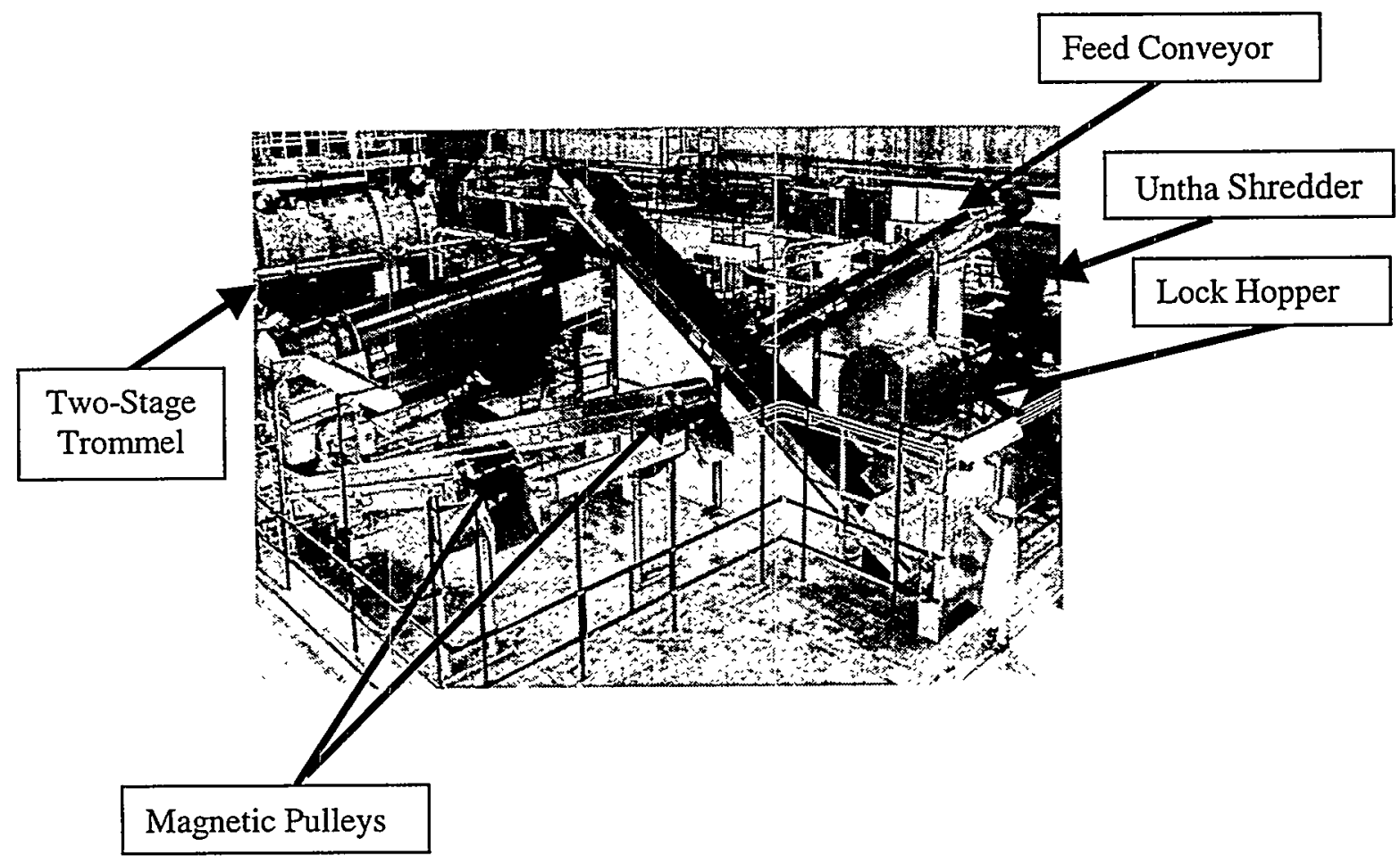

Figure A.1 Argonne Materials Separation Facility 


\section{Appendix B Use of Shredder Residue's Inorganic Fines}

The fines (particles smaller than 1/4 in.) constitute between 30 and $55 \mathrm{wt} \%$ of the ASR mass. The fines are separated as a by-product of the foam and plastics separation process. A typical metallic composition of fines (total and after magnetic separation) is shown in Table B.1. The fines also contain between 20 and $30 \mathrm{wt} \%$ organic material and moisture. The balance is oxygen because the metals are present mostly as oxides. Because these fines are rich in iron and silicon oxides, they might feasibly be used as a source of iron-units in cement production.

Table B.1 Elemental Metal Analysis of the Total Fines and the Magnetic Fraction of the Fines

\begin{tabular}{|lccc}
\hline \multicolumn{1}{|c}{ Element } & $\begin{array}{c}\text { Total Fines } \\
\text { (wt \%) }\end{array}$ & $\begin{array}{c}\text { Magnetic Fines } \\
\text { after One Pass } \\
\text { (wt \%) }\end{array}$ & $\begin{array}{c}\text { Magnetic Fines } \\
\text { after a Second Pass }\end{array}$ \\
\hline Iron & 16.00 & 35.00 & (wt \%) \\
Silicon & 11.00 & 5.00 & 73.80 \\
Calcium & 3.50 & 2.20 & 1.34 \\
Sodium & 2.20 & 0.45 & 0.48 \\
Zinc & 2.00 & 1.60 & 0.24 \\
Aluminum & 1.50 & 0.65 & 0.44 \\
Magnesium & 1.20 & 0.30 & 0.22 \\
Titanium & 0.40 & 0.22 & 0.12 \\
Barium & 0.30 & 0.18 & 0.06 \\
Potassium & 0.25 & 0.11 & 0.15 \\
Lead & 0.25 & 0.19 & 0.05 \\
Manganese & 0.18 & 0.27 & 0.13 \\
Nickel & 0.15 & 0.17 & 0.32 \\
Boron & 0.10 & 0.04 & 0.20 \\
Chromium & 0.07 & 0.09 & 0.01 \\
Copper & 0.05 & 0.05 & 0.08 \\
Zirconium & 0.04 & 0.02 & 0.08 \\
Cobalt & 0.01 & 0.01 & 0.01 \\
Totals & 39.20 & 46.55 & 0.01 \\
\hline Avran & & & 77.82 \\
\hline
\end{tabular}

average of six samples.

${ }^{b}$ One sample was analyzed.

Several cement companies were contacted to see if the fines could be used. Their main concerns regarding the total fine fractions were (1) low iron content, (2) high zinc content, (3) high alkali metals (sodium and potassium) content, and (4) high organics content. The first three affect the properties of the cement, and the fourth may increase air emissions because the iron units are added in the lower temperature zones of the kiln. Therefore, it was necessary to further separate these fines to meet the specifications of the cement makers. 
By using magnetic separation, the fines were separated into a magnetic fraction and a nonmagnetic fraction. The magnetic fraction is, on average, about $40 \%$ of the total fines. Although the iron contents more than doubled, the organic content was still approximately $15 \mathrm{wt} \%$. Subjecting the magnetic fines to a second magnetic separation stage produced a material with less than $5 \mathrm{wt} \%$ organics and less than $0.5 \mathrm{wt} \%$ zinc and lower levels of sodium and potassium. The chemistry of the fines after the second stage of magnetic separation meets the criteria for use as a source of iron units. This fraction constitutes about $35 \%$ of the weight of the total fines.

The composition of the magnetic fraction in general depends upon the moisture level in the ASR. Wet ASR does not separate as cleanly as dry ASR. Therefore, the level of organics and other nonmagnetic material in the magnetic fraction is expected to increase as the moisture content of the ASR increases.

A sample of magnetic fines was TCLP tested, and it passed the test. The TCLP test results are given in Table B.2.

Table B.2 Results of the TCLP Analysis of Magnetic Fines Derived from ASR

\begin{tabular}{|c|c|c|}
\hline Material & $\begin{array}{c}\text { Concentration } \\
\text { (mg/L) }\end{array}$ & $\begin{array}{c}\text { RCRA Max } \\
\text { (mg/L) }\end{array}$ \\
\hline \multicolumn{3}{|l|}{ Metals: } \\
\hline Arsenic & $<0.01$ & 5 \\
\hline Barium & 1.1 & 100 \\
\hline Cadmium & $<0.05$ & 1 \\
\hline Total Chromium & $<0.05$ & 5 \\
\hline \multicolumn{3}{|l|}{ Organics: } \\
\hline Vinyl chloride & $<0.04$ & 0.2 \\
\hline 1,1 Dichloroethene & $<0.100$ & 0.7 \\
\hline Methyl ethyl ketone & $<0.02$ & 200 \\
\hline Chloroform & $<0.04$ & 6 \\
\hline 1,2 Dichloroethane & $<0.04$ & 0.5 \\
\hline Carbon tetrachloride & $<0.04$ & 0.5 \\
\hline Benzene & $<0.06$ & 0.5 \\
\hline Trichloroethene & $<0.04$ & 0.5 \\
\hline Tetrachloroethene & $<0.100$ & 0.7 \\
\hline Chlorobenzene & $<0.04$ & 100 \\
\hline 1,4 Dichlorobenzene & $<0.05$ & 7.5 \\
\hline Pyridine & $<0.5$ & 5 \\
\hline Heptachlor & $<0.0006$ & 0.008 \\
\hline Chlordane & $<0.001$ & 0.03 \\
\hline Methoxy chlor & $<0.007$ & 10 \\
\hline
\end{tabular}


Table B.2 (Cont.)

\begin{tabular}{|c|c|c|}
\hline Material & $\begin{array}{c}\text { Concentration } \\
\text { (mg/L) }\end{array}$ & $\begin{array}{c}\text { RCRA Max } \\
\text { (mg/L) }\end{array}$ \\
\hline \multicolumn{3}{|l|}{ Metals: } \\
\hline Lead & $<0.05$ & 5 \\
\hline Mercury & $<0.01$ & 0.2 \\
\hline Selenium & 0.14 & 1 \\
\hline Silver & $<0.05$ & 5 \\
\hline \multicolumn{3}{|l|}{ Organics: } \\
\hline O Cresol & $<0.03$ & 200 \\
\hline M Cresol & $<0.03$ & 200 \\
\hline P Cresol & $<0.03$ & 200 \\
\hline Hexachloroethane & $<0.07$ & 3 \\
\hline Nitrobenzene & $<0.03$ & 2 \\
\hline Hexachlorobutadiene & $<0.08$ & 0.5 \\
\hline 2,4,5 Trichlorophenol & $<0.04$ & 400 \\
\hline 2,4,6 Trichlorophenol & $<0.03$ & 2 \\
\hline 2,4 Dinitrotoluene & $<0.02$ & 0.13 \\
\hline Hexachlorobenzene & $<0.02$ & 0.13 \\
\hline Pentachlorophenol & $<0.06$ & 100 \\
\hline Lindane & $<0.0005$ & 0.4 \\
\hline Heptachlor epoxide & $<0.08$ & 0.008 \\
\hline Endrin & $<0.003$ & 0.02 \\
\hline Toxaphene & $<0.05$ & 0.5 \\
\hline
\end{tabular}


$\Delta$

B-4 


\section{Appendix C Selective Recovery of Thermoplastics by Using Froth Flotation}

In a related program that recovers plastics from obsolete home appliances, a froth flotation process was developed and pilot tested to separate ABS and high-impact polystyrene (HIPS). The waste stream is first granulated to about $1 / 4$-in. pieces, and then the light materials (rigid foam insulation, polyethylene, and polypropylene) are separated by conventional sink/float methods. The heavier materials (PVC, nylons, and residual metals) are also separated by using conventional sink float techniques. This leaves a middling fraction that is essentially the $A B S$ and the HIPS material. The Argonne froth flotation process has been pilot tested and was shown to be capable of separating the ABS and the HIPS from this mixture in purities greater than $99 \%$ and yields greater than $80 \%$.

Argonne conducted limited testing to extend this process to ASR plastics. The following comments and observations are in order:

- The first step in recovering the plastics was to identify the plastics that are present in the largest quantities and have the highest market value. The results are presented in Table C.1, for cars and appliances built in 1985.

Table C.1 Values and Quantities of the Major Plastics in ASR

\begin{tabular}{|c|c|c|c|}
\hline Plastic Type & $\begin{array}{c}\text { Plastics in ASR } \\
\left(10^{6} \mathrm{lb}\right)^{\mathrm{a}}\end{array}$ & $\begin{array}{c}\text { Fraction of } \\
\text { Total Plastics } \\
\text { in ASR }\end{array}$ & $\begin{array}{c}\text { Plastics Value } \\
\qquad\left(10^{6}\right)\end{array}$ \\
\hline Polypropylene & 455 & 0.144 & 107 \\
\hline ABS & 434 & 0.138 & 193 \\
\hline PVC & 366 & 0.117 & 77 \\
\hline Flexible foam & 360 & 0.114 & 192 \\
\hline Nylons & 208 & 0.066 & 155 \\
\hline Polyethylene & 186 & 0.059 & 49 \\
\hline Polyester TP & 84 & 0.027 & 50 \\
\hline Polycarbonates & 73 & 0.023 & 77 \\
\hline High-impact polystyrene ${ }^{b}$ & 73 & 0.023 & 16 \\
\hline
\end{tabular}

${ }^{a}$ Assuming $100 \%$ of automobiles and $60 \%$ of appliances are shredded.

b The quantity of HIPS in ASR is small. However, when refrigerators are shredded separately, it will be the second largest component in the waste stream.

- Plastics in ASR are greater in number than in the reject material from shredding one item (such as refrigerators) or related items (such as home appliances). ASR plastics include plastics from refrigerators and all other home appliances, in addition to the automotive plastics. The list includes, in descending order of concentration, polypropylene, ABS, PVC, polyurethane foam, polyester thermoset, nylons, polyethylenes, other urethanes, PPO styrene, polyester thermoplastics, SMA, HIPS, 
polyurethane rigid foam, polycarbonates, acrylics, phenolics, PP EPDM modified, acetals, PC PBT, ionomers, butyrals, SAN, ABS/PC alloys, PPO nylons, epoxies, polyester elastomers, and alkydes. Many others are also present in smaller quantities, and new ones are added to the list every year. Obviously, as the number of species in the mixture increases, the difficulty in identifying froth flotation solutions and conditions that are selective toward only one of the mixture components increases. Therefore, multiple concentration and frothing steps will be required to produce quality material. The growth in the use of major automotive plastics is shown in Figure C.1.

- Plastics in ASR contain a higher level of surface contamination than the plastics in waste streams generated by shredding refrigerators and home appliances. Because froth flotation is, to a large extent, a surface phenomenon, the surfaces need to be cleaned. Water and surfactants can be used for cleaning. The resulting wastewater is not likely to be a hazardous waste.

- The plastics mixture must be granulated to a size of about $1 / 4$ in. The granulation should be done after sink/float techniques are applied to concentrate the targeted plastics and remove the metals.

Results obtained so far indicate that froth flotation is a very promising technique for separating durable plastics from appliance and automotive shredder waste streams. Argonne has successfully tested, at the bench scale, a process to separate PP from a mixed-plastics fraction that was floated in water at a specific gravity of 103 by using a solution having known specific gravity, $\mathrm{pH}$, and surface tension. The PP concentration in the floating material was greater than $99 \%$. The PP yield was about $65 \%$. An invention disclosure for this process has been prepared.

Froth flotation experiments were conducted to recover ABS, PC, and ABS/PC materials from unused, shredded 1997 instrument panel shells. The ABS, PC, and ABS/PC materials were recovered at purity levels in the upper $90 \%$ range and with a yield of over $90 \%$. However, when we applied the same conditions to materials derived from used, shredded complete instrument panels that were removed from scrapped automobiles, the purities and the yields were in the $80 \%$ range. Similarly, lower yields $(\sim 50 \%)$ and high purities $(\sim 98 \%)$ were observed when the same conditions were applied to a mixture of "white plastics" that were handpicked from ASR. Nevertheless, the results are encouraging and indicate that higher yields and purities can be achieved when the conditions are properly modified and optimized.

The history of the plastic material is expected to affect its behavior in the froth flotation solution. This change in behavior may be due to a number of factors, including the following:

- Presence of "oils" and other contaminants on the surface that will highly affect the hydrophilicity/hydrophobicity of the material.

- Surface composition might have changed over the years because of oxidation reactions and exposure to UV light. 


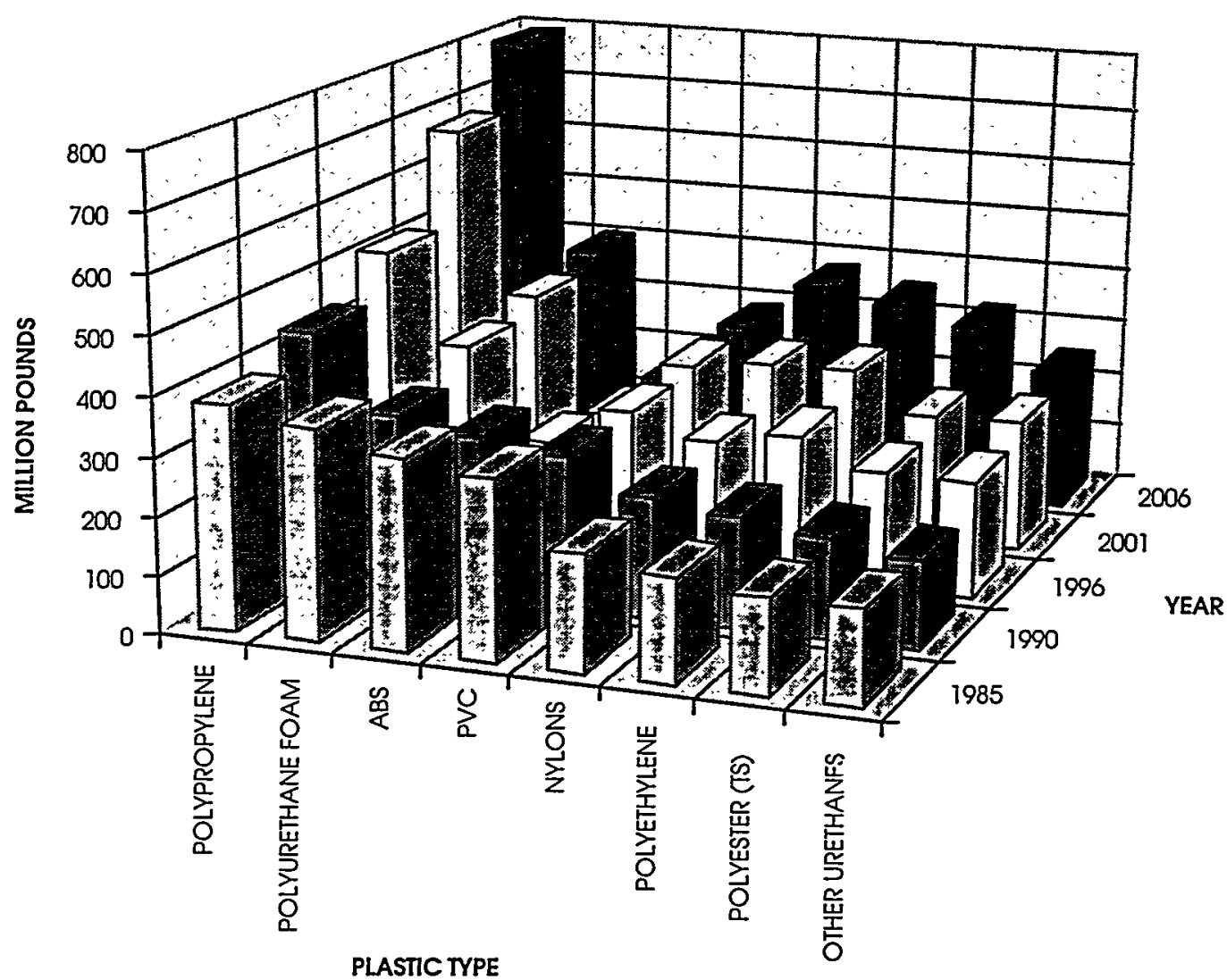

Figure C.1 Growth in Major Automotive Plastics

- Surface roughness is likely to change upon shredding and handling in mechanical systems, which may result in uneven distribution of the surface forces over the surface of the plastic chips.

- Different grades and formulations of a given plastic material have been produced over the years.

Therefore, such a surface is expected to behave differently from that of the corresponding virgin pellets made today. It is important to conduct experiments with the actual material that is to be separated before attempting large-scale production runs.

Separation of ASR plastics using froth flotation could potentially be economically attractive. It is premature at this stage to quantitatively estimate the production cost of the various plastics. However, it appears that for the high-value and large-volume engineered 
materials, such as the ABS, PC, and ABS/PC alloys, froth flotation will be economical. Polypropylene is also an attractive plastic to pursue because it is the largest plastic fraction in ASR, and its use is expected to continue to increase (Figure C.1). In addition, separation of the light olefinic fraction is required as part of concentrating the other plastics. 


\section{Appendix D Recycled Foam Material Information Sheet}

NOTE: The following information follows the format of a Material Safety Data Sheet (MSDS) and was compiled for polyurethane foam recovered from auto shredder residue. It is not, however, intended to be an MSDS.

\section{COMPOSITION/INGREDIENTS}

Components:

Chemical Formula: several types of flexible polyurethane foam. Synonyms: Polyfoam sponge, Polyfoam plastic sponge, Etheron Sponge, Polyurethane ester foam, polyurethane ether foam, Polyurethane sponge, foam rubber. It may contain low levels of PCBs, Silica, metal fines.

\section{HEALTH /SAFETY}

Primary routes of entry: Inhalation

Acute health hazards: Ingestion: May cause choking.

Inhalation: Dust generated during cutting can be a nuisance.

Skin contact: May cause mechanical skin irritation.

Eye contact: The dust may cause eye irritation.

\section{FIRST AWD MEASURES}

Emergency first aid procedures:

Eye contact: Flush eyes with potable water for at least 15 minutes.

Skin contact: Wash skin with water and soap.

Inhalation: Remove to a fresh air environment.

Ingestion: May cause choking if ingested. Call a physician immediately. Remove foam from mouth and throat. Have patient checked by a physician for any residual foam in the throat and esophagus. 


\section{FIRE-FIGHTING MEASURES}

Flash point

Extinguisher media:

Special fire

fighting procedures:

Unusual fire and explosion hazards: $>200^{\circ} \mathrm{C}$

Carbon dioxide and dry chemicals. Water should not be used because at high temperatures, it results in the hydrolysis of the foam and the hydrolysis products include polyols and isocyanates.

When heated to decomposition, or in the case of fire, it emits acrid smoke and fumes of $\mathrm{CN}^{-}$and $\mathrm{NO}_{x}$. Wear NOISH/MSHAapproved, self-contained breathing apparatus to prevent breathing of $\mathrm{CN}$ and $\mathrm{NO}_{\mathrm{x}}$ and full protective equipment.

Foam can emit combustible gases, making flashback possible. It also burns with high-temperature heat.

Material is a solid; sweep up, no need for special precautions.

Can be disposed of in regular landfills in accordance with local, state, and federal regulations.

Notify the local fire companies of the presence of large quantities of foam.

\section{HANDLING AND STORAGE}

Handling procedures: It may contain small pieces of wire, metals, or glass.

Storage:

Store in a dry environment at ambient conditions and away from heat and open flames. Also, keep the material away from strong oxidizing agents.

Other precautions:

Notify local fire companies of the presence of large quantities of foam. 


\section{EXPOSURE CONTROLS AND PERSONAL PROTECTION}

Ventilation:

Respiratory protection:

Protective gloves:

Eye Protection:

Protective clothing:

Hygienic work practices:
No special requirements.

Dust masks are recommended for general handling. Use NIOSH/MSHA-approved organic cartridges when cutting the foam using hot wires.

Use impervious puncture-resistant gloves.

ANSI-approved Chem workers goggles.

None required.

None required.

\section{STABILITY AND REACTIVITY}

Stability:

Incompatibility:

Conditions to avoid:

Hazardous decomposition products:
Stable under normal pressure.

Reacts with steam at elevated temperatures and produces isocyantes. It is also attacked by strong oxidizing agents.

Temperatures of over $300^{\circ} \mathrm{F}$.

Polyols, isocyanates, $\mathrm{NO}_{x}$, and $\mathrm{CN}^{*}$. 
$\Delta$

D-4 


\section{U.S. Patent: Efficient Continuous Dryer for Flexible Polyurethane Foam and Cleaning Apparatus}

\section{United States Patent} Jody et al.

[54] EFFICIENT CONTINUOUS DRYER FOR FLEXIBLE POLYURETHANE FOAM AND CLEANING APPARATUS

[75] Inventors: Bassam Jody. Chicago; Edward Daniels. Oak Lawn; Joseph A. Libera. Clarendon Hills. all of $\mathrm{II}$.

[73] Assignee: The University of Chicago. Chicago. III.

[21] Appl. No.: 692,801

[22] Filed:

Jul. 30, 1996

[51] Int. $\mathrm{Cl}^{6}$

[52] U.S. Cl. B08B 3/00: B08B 1/02 134/26; 134/21; 134/32:

$134 / 30$

[58] Field of Search 134/26. 21. 32. $134 / 30$
References Cited

U.S. PATENT DOCUMENTS
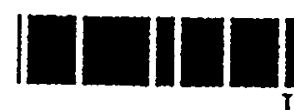

USO05882432A

[11] Patent Number:

$5,882,432$

[45] Date of Patent:

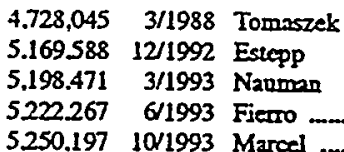

210787

Primary Examiner-Ponnathapura Achutamurthy Assistant Examiner-P. Ponnaluri Atromey, Agent, or Firm-Emrich \& Dithmar

[57]

ABSTRACT

A method of cleaning poiyurethane foams where the material is transported through a wash station while alternately soaking the polyurethane foam in an organic solvent and squeezing solvent from the polyurethane foam a number of times. Then the polyurethane foam is sent through a rinse or solvent transfer station for reducing the concentration of solvent in the foam. The rinsed polyurechane foam is sent to a drying station wherein the foam is repeatedly squeezed while being exposed to hot air to remove wer air from the foam. 


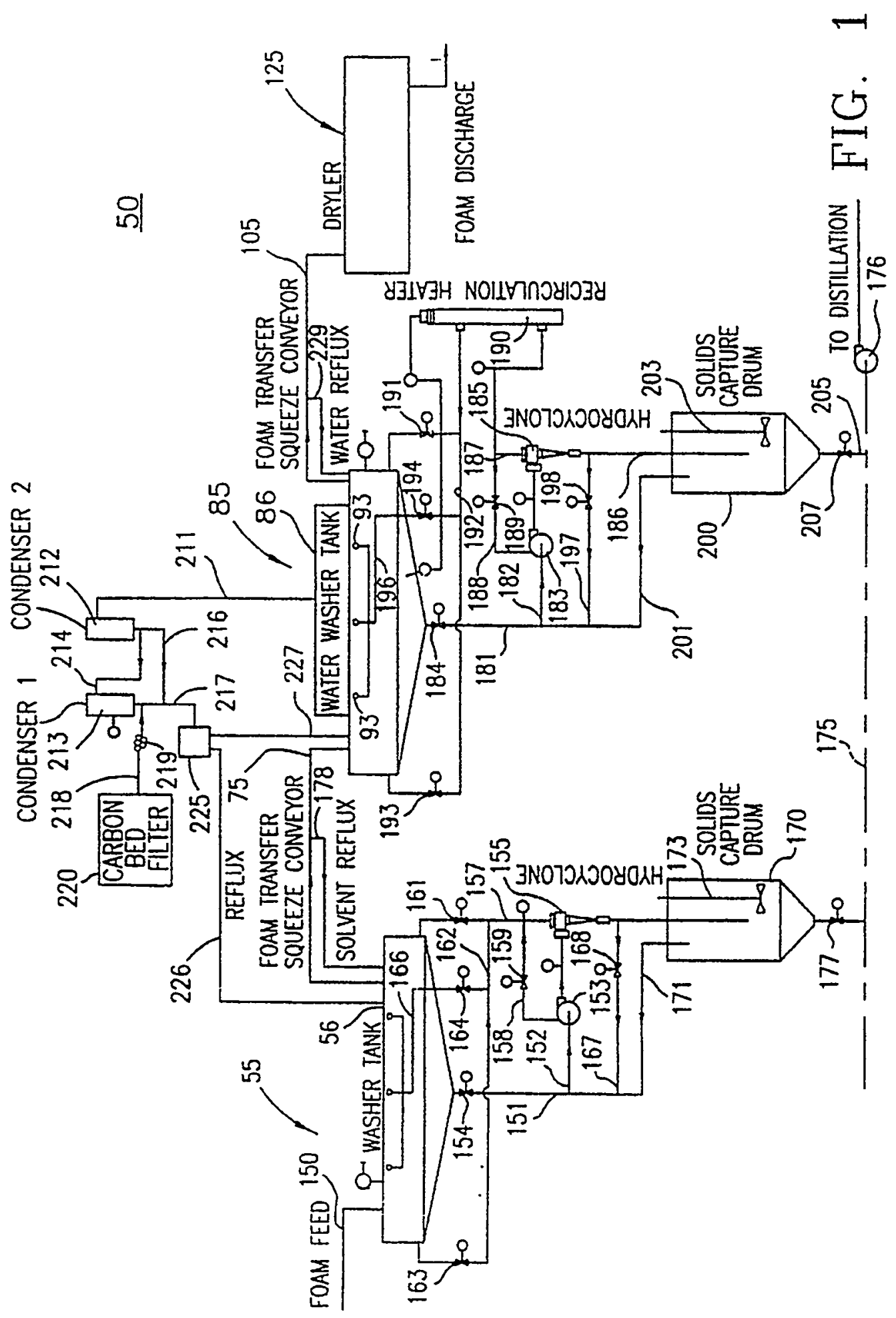


Sheet 2 of 4

$\mathbf{5 , 8 8 2 , 4 3 2}$

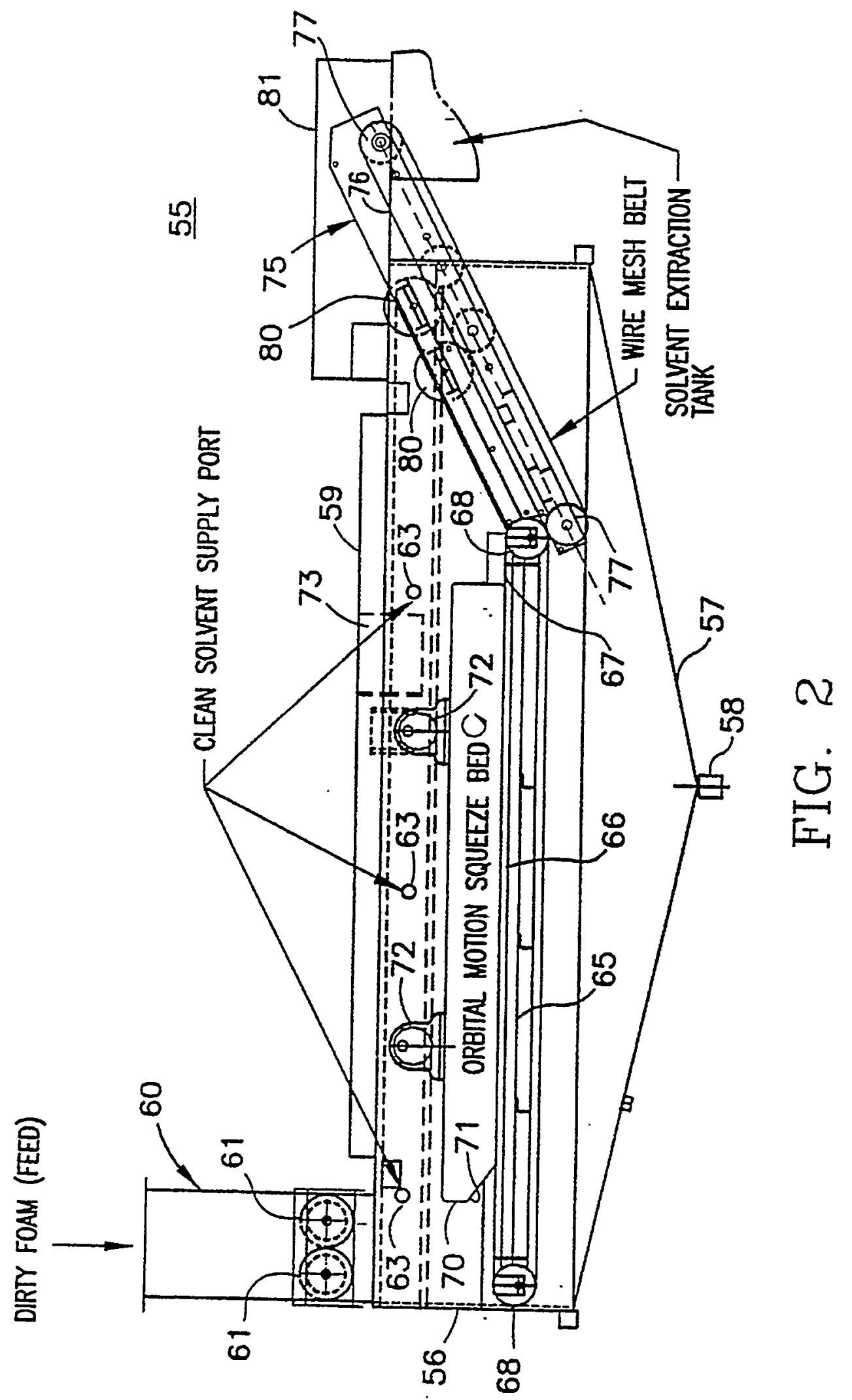




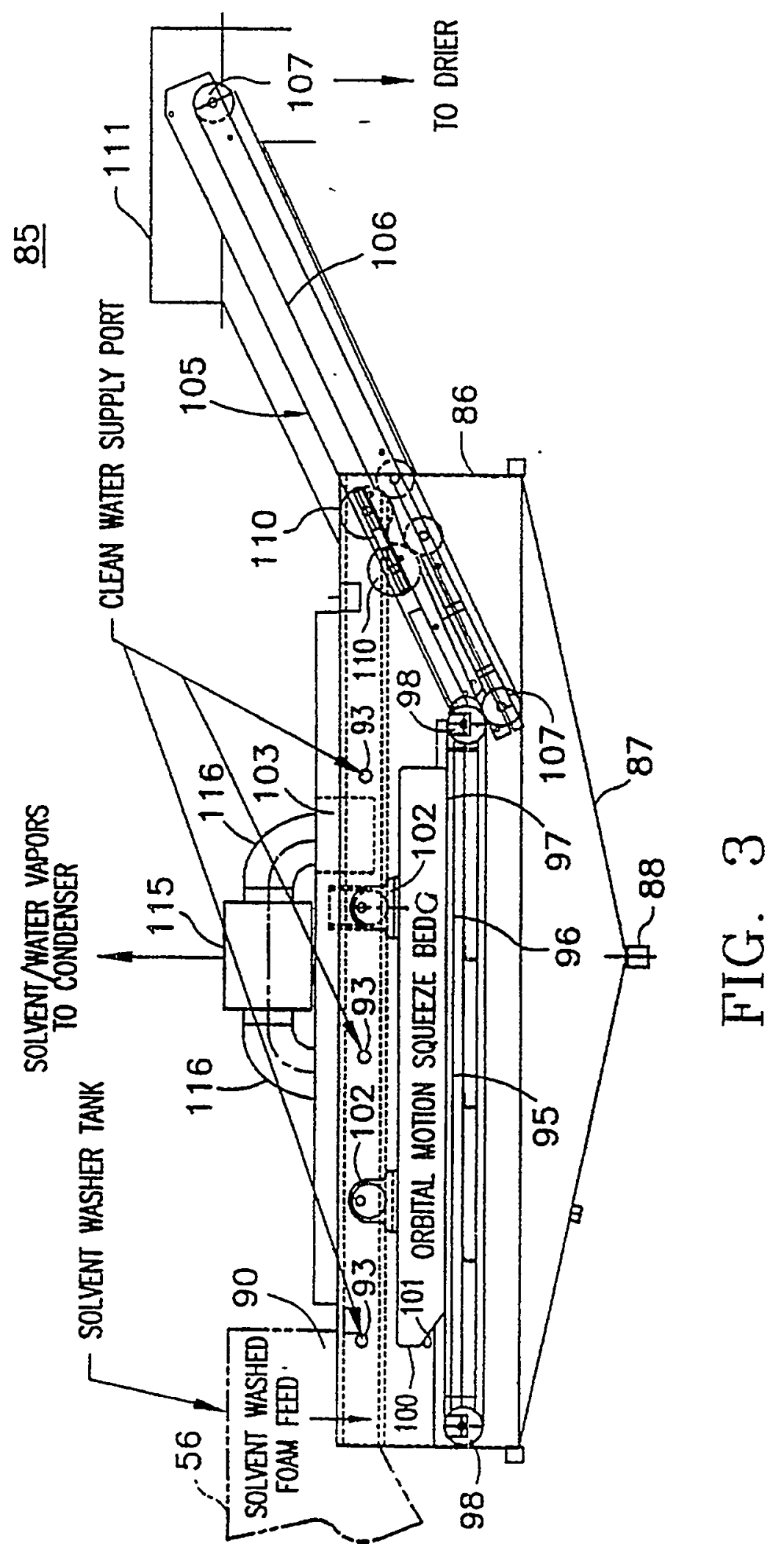




\section{U.S. Patent}

Mar. 16, 1999

Sheet 4 of 4

$5,882,432$

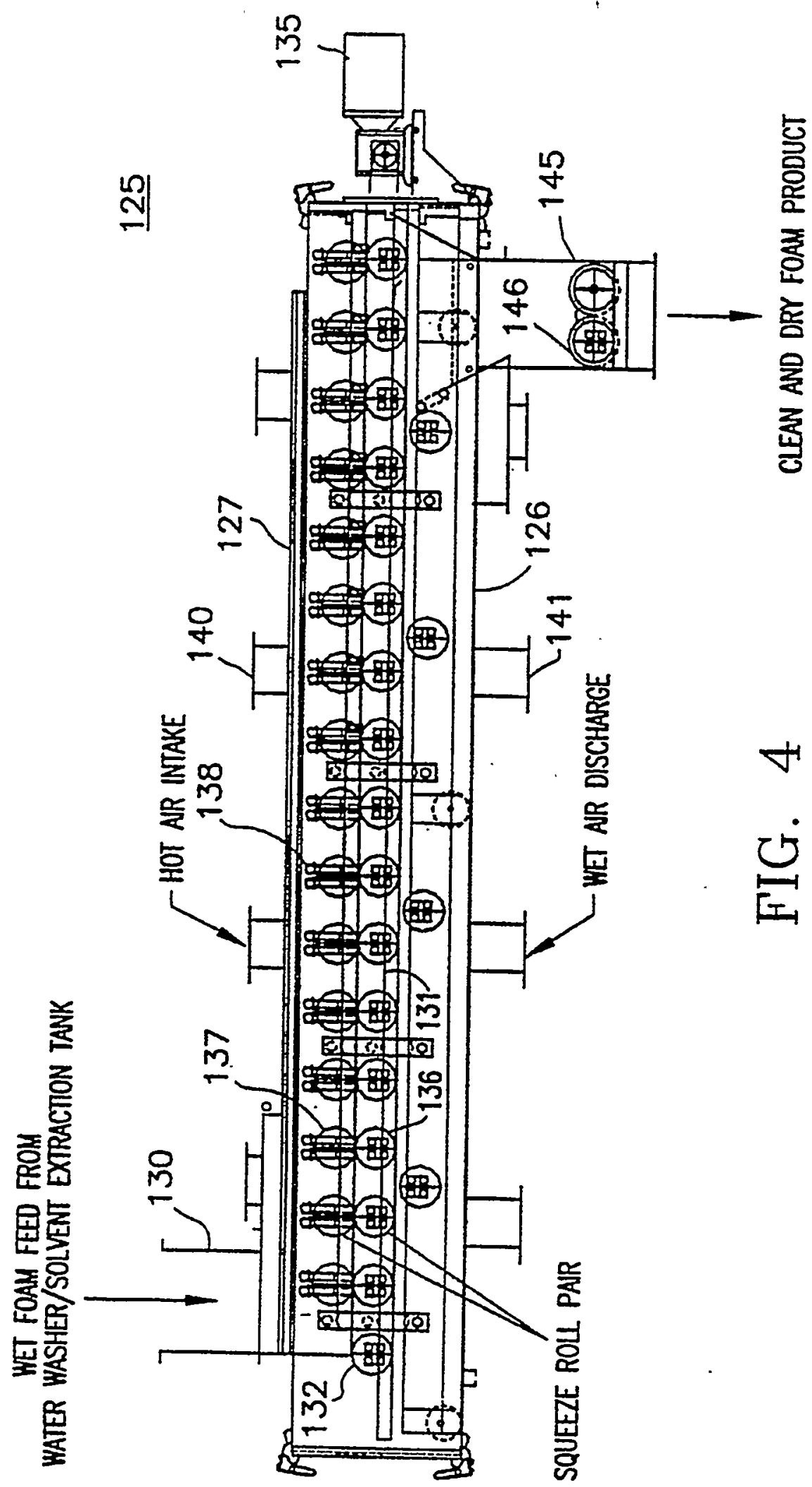


1

\section{EFFICIENT CONTINUOUS DRYER FOR FLEXIBLE POLYURETHANE FOAM AND CLEANING APPARATUS}

CONTRACTUAL ORIGIN OF THE INVENTION

The United States Government has rights in this invention pursuant to Contract No. W-31-109-ENG-38 between the U.S. Department of Energy and The University of Chicago representing Argonne National Laboratory.

\section{BACKGROUND OF THE INVENTION}

This invention relates to a continuous process for cleaning and drying flexible porous materials such as foam and more paricularly. polyurethane foam recovered from foam containing waste streams such as automobile shredder residue. Contaminants in the foam primarily are oils and other organies as well as dirt.

Specifically. the invention relates to apparatus and system for processing the foam through a series of devices which repeatedly squeeze and release the foam to allow solvent in a washing apparatus to infiltrate the pore structure of the 20 foam and then be mechanically squeezed therefrom a plurality of times.

In addition to which the invention inciudes apparatus for rinsing the cleaned foam to rid the foam of the organic solvents and thereafter to dry the foam also with repeated mechanical squeezing of the foam to reduce the amount of time the foam resides in the solvent transfer station (referred to here sometimes as a rinse station) and the dryer. Complete flow processes are disclosed for recycling and treating various waste streams from the washing station. the rinsing 30 station. and the drying station.

\section{SUMMARY OF THE INVENTION}

A principal object of the present invention is to provide a system for removing organic oils. greases and inorganic dirt 35 from foam like materials and more particularly. from polyurethane foam from auromobile shredder residue.

Another object of the invention is to provide a system including a washing station. a rinsing or solvent transfer station and a drying station in which the resident time of the 40 foam in any station is shor.

Yet another object of the present invention is to provide a system of the type set forth in which the organic solvent and water in the system are recovered and recycled for continu. ous use. thereby minimizing emissions to the environment.

The invention consists of certain novel features and a combination of parts hereinafter fully described. illustrated in the accompanying drawings. and particularty pointed out in the appended claims. it being understood that various changes in the details may be made without departing from the spirit. or sacrificing any of the advantages of the present invention.

\section{BRIEF DESCRIPIION OF THE DRAWINGS}

FIG. 1 is a schematic process flow diagram showing the 5 system. apparatus and method of the present invention:

FIG. 2 is a schematic representation of the washing station of the present invention:

FIG. 3 is a schematic representation of the rinsing or solvent transfer station of the present invention: and

FIG. 4 is a schematic representation of the drying station of the present invention.

\section{DETAIIED DESCRIPTION OF THE PREFERRED EMBODIMENT}

Referring now to the drawings. there is disclosed in FIG. 1 a foam treament process 50 which includes a washing
2

station 55. a solvent transfer station 85 and a drying station 125. More particularly. by reference to FIG. 2. the washing station 55 includes a tank 56 which has a sloping botzom portion 57 leading to an outlet $\mathbf{5 8}$. The tank $\mathbf{5 6}$ is enclosed by a cover 59 which is provided with an iniet chute 60 through which dirty foam is fed into the washing station 55. A pair of rollers 61 is positioned within the chute 60 to provide both feeding mechanism as well as a vapor lock mechanism for the washing station 55 . thereby to prevent the

10 escape of organic solvent vapors from the station 55. A plurality of clean solvent supply ports 63 spaced axially of the longitudinally extending washing station 55 are provided to introduce clean solvent into the washing station. The solvent can also be supplied in a countertlow made with 5 respect to the foam to increase the dirt loading in the solvent and reduce the rate at which the solvent is regenerated.

Inside the washing station $\mathbf{5 5}$ is a longitudinally extending support bed 65 which includes a perforate base plate 66 which may be made of any suitable material such as Tefion or stainless steel on which is supported a mesh conveyor bett 67 extending around pulleys 68 positioned one at the inlet end of the washing station 55 and the other toward the outlet end of the washing station.

An orbital motion bed or plate 70 having a chamfered inlet surface 71 is supported on a pair of eccentric drives 72 . one of which is provided with an eccentric drive motor 73 . As disciosed in FIG. 2. the crbital motion bed 70 rotates in a counterciock wise direction so as to move foam from the inlet chute 60 thereof toward the outlet end of the washing station 55 . This forward motion is promoted by the slippage at the wire mesh idling belt due to the forward shear force established through the foam by the bed. An outlet foed conveyor 75 is located at the outlet end of the washing station 55 and is provided with a conveyor belt 76 positioned around a pair of pulleys 77 which receives pieces of polyurethane foam from the conveyor belt 67 and transports same angularly upwardly out of the washing station 55 . A pair of squeeze rollers 80 positioned intermediate the palleys 77 physically compress the foam as it leaves the wasting station 55 so as to express fluid such that the foam leaving the washing station 55 has absorbed therein fluid generally not more than about its own weight.

Finally, a cover 81 is positioned over the outlet partion of the station 55 cooperating with the cover 59 entirely to close the washing station 55 to prevent the escape of organic solvent vapors into the atmosphere.

Solvents suitable for use in the washing station 55 are perchloroethylene. tetrachloroethylene. various alcohols. acetone. hexane. and various mixtures thereof including "green solvents" (that is biodegradeable solvents) along with -other organic solvents which are suitable to dissolve automobile oils and greases normally found in polyurethane foarn from automobile shredder residue. Although the invention is described with reference to the preferred organic solvent perchloroethylene. it is not meant to be limiting in any way whatsoever. but merely for illustrative purposes only. In addition. the reciprocating bed is one way to squeeze the foam. Other forms including rollers and pressure can be $\infty$ used to accomplish the same objective. This is also true in the case of the solvent exchange bed described below.

Referring now to FIG. 3. there is disclosed the solvent transfer station 85 which includes a solvent extraction tank 86 provided with a sloping bortom 87 and an outlet 88 . A 65 cover 89 covers the majority of the tank 86 . The tank 86 may be similar or identical in most respects to the tank 56. An inlet 90 receives foam from the washing station 55 and 
3

introduces the foam segments into the solvent transfer or rinse station. A plurality of clean water supply ports 93 feed water into the extraction tank 86 to a level which covers a support bed 95 positioned below a perforated base plate 96 also below a mesh conveyor belt 97 positioned around a pair of pulleys 98. The configuration is similar to that previousiy described for the washing station 55 .

An orbital bed 100 is positioned slightly above the conveyor belt 97 . the orbital bed having a chamfered inlet surface 101 and a pair of eccentric drives 102. one of which being connected to an eccentric drive motor 103 . The orbital motion of the bed 100 is counterclock wise as is the orbital motion of the bed 70. both of which move downwardiy and to the right as viewed in the drawings in order to compress polyurethane foam segments which are positioned between the bed and the adjacent conveyor belt and support bed underneath same.

An outfeed conveyor 105 is located at the end of the tank 86 anay from the inlet 90 and includes a conveyor belt 106 positioned around a pair of pulleys 107 . Intermediate the pulleys 107 are squeeze rollers 110 which cooperate to express water from the polyurethane foam being transported on the conveyor 106 so that the foam contains water not in excess of about its own weight as it leaves the rinsing station 85. A cover 111 is positioned over the conveyor 105 to enclose the solvent transfer or rinse station 85 in the same manner that the washing station $\mathbf{5 5}$ is enclosed. thereby to prevent the evaporation of any organic solvent to the atmosphere. Vapors such as steam and evaporated solvent are conducted out of the solvent transfer or rinse station 85 by means of a vapor duct 115 connected to the top of cover 89 on the tank 86 by means of a pair of conduits 116. the vapor duct 115 being connected to an exhaust fan 219 (as will be described). so that the rinsing station 85 as well as the washing station $\mathbf{5 5}$ are operated under negative pressure. Also not shown: vapors are condensed and scparated. Solvent refiuxes to the solvent tank and water refluxes to the water tank

The drying station 125 is more particulaty sbown in FIG. 4 and inciudes an clongared chamber 126 provided with an enclosure. 127. An inlet chute 130 directs foam segments from the rinsing station 85 to a conveyoc belt 131 extending axially of the drying station 125. the belt being supparted by a pair of pulleys 132. One of which is connected to a motor 135 for transporting the conveyor belt 131 in a clockwise direction thereby to move the polyurechane foam segments from the inlet end of the dryer $\mathbf{1 2 5}$ to the outlet end of the dryer.

A plurality of transversely extending axially spaced apart bottom rollers 136 are positioned below the upper flighs of 50 the conveyor belt $\mathbf{1 3 1}$ and cooperate with a plurality of rollers 137 in registry with selected ones of the bottom rollers 136 . The top rollers 137 are spring loaded as at 138 to provide a plurality of compressions to the polyurethane foam being transported on the conveyor belt 131. Actually. ss the springs provide relief against incompressible contaminants such as metal tramp. Normally, fixed rollers compress foam adequately. Springs are for safety. and the gap berween the upper roller and belt is typically $i_{x}$ inch. and can be adjusted. Each compression of the polyurethane foam as it passes through the pairs of rollers 136.137 causes hot moist air to be expressed from the foam to be replaced intermediate the rollers by hot dry air as the foam expands. While being squeezed. the hot belt roller aids in evaporation by direct heat conduction. The succession of squeezing steps facilitates the drying of the foam such that the foam can be dried in less than about 15 minutes of residence time in the

\section{4}

drying station 125. Air is introduced into the drying station 125 through a plurality of hot air inlets 140 and hot air is removed from the drying station 125 by a plurality of wet air discharge conduits 141. The movement of air in the drying station 125 is in a cross countercurrent mode relative to the foam moving through the drying station from the left to the right as viewed in the drawing. The combination of cross current and countercurrent flow enhances the drying effciency in the drying station 125.

10 An outlet chute 145 is located at the right hand end of the drying station 125 and includes a pair of vapor lock rollers 146 preventing cold air contamination.

Operation of the system will now be explained by reference particularly to FIG. 1 of the drawings.

Referring now to FIG. 1. there is seen that segments of polyurethane foam are fed via a conveyor or other suitable mechanism 150 into the wash station 55. The wash station 55 has suitable organic solvent therein such as perc maintained at a liquid level in the tank 56 so as to cover the bed 70 at its highest point. The orbital bed 70 is operated such that segments of foam passing between the bed and the support bed 65 are squeezed between about 20 and about 100 times during the transportation through the wash station 55. There is a direct relationship between the geometry of the eccentric drive and the forward progress stroke. The present design achieves about 1 inch of forwand motion stroke. The frequency does not affect the number of squeezes. but does change the residence time. The periodic squeezing of the foam serves to enhance the dissolution of any grease and oil in the foam as well as dislodging inorganic dirt and grit from the foam. all of which collects at the bottom of the tank 56. The squeczing results also in the discharging of the dirt loaded solvent out of the foam to be replaced with cleaner solvent and thus enhances the cleaning efficiency. The dirty solvent. dirt and grit exits the tank 56 through an outlet line 151 which brancies as at line 152 to a pump 153. the pump 153 serves to transport dirty solvent and solids into a hydrocyclone 155. A valve 154 is in the line 151 serves to control when solvent is removed from the tank 56 through the outlet 58 thereof.

The hydrocyclone 155 operates in the same manneras any other hydrocyclone and is provided with a solid outlet 156 and an overhead line 157. The overhead line 157 branches into a line 158 controlled by a valve 159 which recycies to the iniet end of the pump 153 to provide recycte ability of the overhead from the hydrocyclone 155 . Valves 161 and 163 are used to wash down the bottom of the tank 56 to rid the tank of accumulated dirt and sludge.

Accordingly. it is seen that cleaned perc or other solvent. if used. from the hydrocyclone 155 . can be recycled to the tank 56 at a variety of locations to provide cleaner solvent to the foam segments as they enter the tank 56 . as they proceed through the tank 56 and as they are exiting the tank 56. The hydrocyclone 155 extracts dir. but not soluble components. The latter is controlled by continuous distillation.

A line 156 from the bottom of the hydrocyclone $\mathbf{1 5 5}$ is branched as at 167 and controlled by valve 168 to recycle the 60 bottoms from the hydrocyclone back through the purmp 153. and hence. into the hydrocyclone again for further separation. If the valve 168 is closed. then the bottoms from the hydrocyclone $\mathbf{1 5 5}$ are transported to a solid carch or drum 170 which is provided with an overhead line 171 which 65 leads to the pump 153 via the line 152 . The solid eatch or drum 170 is also provided with a stirer 173 or agitator so that the material in the drums is agitated during the removal 


\subsection{2 .432}

5

of same through the outlet line $\mathbf{1 7 5}$ controlled by a valve 177. A pump 176 pulls and transports the bottoms from the drum 170 to a distillation facility. not shown.

Finally. a perc reflux line $\mathbf{1 7 8}$ is provided from the cover 181 of the washing station 55 back to the tank 56 so that perc squeezed from the foam during the transfer thereof from the washing station $\mathbf{5 5}$ to the rinsing or solvent transfer station 85 by passage through the squeeze rolls 80 can be recycled into the tank 56 and not be wasted.

Still referring to FIG. 1 of the drawings. the rinse or solvent transfer station 85 is provided with a dirty water outlet line 181 connectod to the discharge port 88 which leads through a line 182 to a pump 183 for transportation the dirty water from the rinsing or solvent transfer station to a hydrocyclone 185. A valve 184 is positioned in the line 181 intermediate the discharge port 88 and the pump 183 to control the discharge cycles from the rinse station 85 . As may be imagined. some of the warer in the rinsing station 85 is contaminated with organic solvent and grit or dint dragged over from the washing station 55 by the residual liquid in the foam segments transported into the wash station through the inlet chute 90. Technically. the water steam strips the solvent and an additional function of the water is to dissolve water soluble dirt remaining in the foam.

The hydrocyclone $\mathbf{1 8 5}$ is similar in construction to the hydrocyclone 155 and is provided with a bottoms outlet 186 and an overhead line 187. The overtead line 187 leads to a recycle line 188 controlled by a valve 189 . the recycle line 188 feeding to the inlet end of the pump 183. Valves 191 and 193 are used in wash down purposes as previously described.

Valves 193 and 194 control the recirculation of hot water from the heater 190 to the rinse tank 86 . the hot water in the tank 186 generally being maintained in the range of from about $85^{\circ} \mathrm{C}$ to about $100^{\circ} \mathrm{C}$. for optinum results. Water temperantres in excess of $100^{\circ} \mathrm{C}$. causes too much water to evaporate while temperatures less than about $85^{\circ} \mathrm{C}$. require the foam to be in the rinse station 85 longer than the desired 15 minutes. In general. the foam is present in the rinse station in the range of from about 3 to about 15 minutes which is the same as the residence time of the polyurethane foam in the wash station.

The hydrocyclone 185 with the bottoms or solids outlet 186 is also provided with a recycle line 197 with a control valve 198 to recycle a partion of the outlet from the hydrocyclone 185 . the bottoms 186 leading to a solids capture drum 200 similar to the drum 170. An overtead line 201 leads to the recycle circuit previously described while a stirrer or agitator 203 is provided in the drum 200 so that when the valve 207 is opened and the outles line 205 is activated. liquids as well as solids leave the drum 200 and are transported through the line $\mathbf{1 7 5}$ and the pump 176 to a distillation facility. not shown.

The rinsing station 85 and more particularly the tank 86 is provided with an overhead outlet 211 connected to the vapor duct 115. the line 211 leading to a pair of condensers 212 and 213 interconnected by a line 214 . An ourlet line 216 from the condenser 212 joins an outlet line 217 from the condenser 213 and leads to a decanter 225 which separates any perc from the water and recycles the perc through line 226 to the washing station 55 and the water through line 227 to the rinse station 85. Another line 218 from condenser 213 controlled by exhaust fan 219 transmitting vapor such as non-condensible air with residual perc and water to a carbon bed filter 220 for final cleaning.

Finally. a water reflux line 229 is provided in the outlet conveyor 105 from the rinse station 85 to the dryer 125

\section{6}

thereby to conserve water and to recycle same as it is squeezed from the foam as the foam leaves the rinse station 85.

The foam leaving the rinse station 85 is transported to the dryer through the inlet 130 thereof where it encounters hot air preferably maintained in the range of from about $100^{\circ} \mathrm{C}$. to about $150^{\circ} \mathrm{C} .150^{\circ} \mathrm{C}$. is lower than the decomposition temperature of the preferred solvent pere and also lower than the degradation temperature of the polyurethane foam com10 monly found in automobile shredder residue. If the system is used for different foams or with different solvents the drying temperature may be higher or lower. but in any case it is important that the maximum temperature for the hot air be less than the lower of the decomposition termperanure of 15 the solvent and the degradation temperature of the foam. In general. the residence time of the foam in the drying station 125 is the same as in the wash station $\mathbf{5 5}$ and the rinse station 85 and that is between about 3 and about 15 minutes.

The orbital beds 70 and 100. respectively in the washing station 55 and the rinsing station 85 . are operated to provide about 20 and about 100 squeezes of the foam as it passes along the respective conveyor. The conveyors are preferably not driven. The index in response to shear force transmitted through the foam as the bed moves to the right in the bottom portion of the stroke. It bas been found that this number of squeezes significantly enhances the washing efficiency in the wash station 55 and the rinsing efficiency in the rinse station 85.

Because all three pieces of equipment are completely covered. and the system is operated at negative pressure. little if any organic solvent evaporates into the atmosphere. The vapor from the condensers 212 and 213 is transmitted via a line 218 and a pump 219 to a carbon filter bed 220 35 thereby to prevent the discharge of any organic vapor to the atmosphere.

As can be seen. therefore. the system $\mathbf{5 0}$ described is a completely closed so that the fluids and the vapors from the system are always used to the maximum amount with the 40 only material being disctarged from the solid capture drums 170 and 200.

While there has been disclosed what is considered to be the preferred embodiment of the present invention. it is understood that various changes in the details may be made without departing from the spirit. or sacrificing any of the advantages of the present invention. These changes include but are not limited to the use of different solvents. various ways of squeczing the foam and different methods of so moving the foam through the beds.

The embodiments of the invention in which an exciusive property or privilege is claimed are defined as follows:

1. A method of cleaning polyurethane foam. comprising transporting polyurethane foam from a source thereof 55 through a wash station while alternately soaking the polyurethane foam in a liquid bath of an organic solvent and squeezing solvent from the polyurethane foam a plurality of times. transporting the polyurethane foam through a rinse station. transporing the rinsed polyurethane foam to a to drying station wherein the foam is repeatedly squeezed while being exposed to hot air. and continuously removing wet air from the drying station.

2. The method of claim 1. wherein the organic solvent is perchloroethylene.

65 3. The method of claim 1. wherein the polyurethane foam is squeezed between about 20 and about 100 times during transportation through the wash station. 
7

4. The method of claim 1 . wherein the polyurethane foam is maintained in the wash station from about 3 minutes to about 15 minutes.

5. The method of claim 1. wherein the foam is rinsed with water in the rinse station.

6. The method of claim 5. wherein the water in the rinse station is maintained at a temperature in the range of from about $85^{\circ} \mathrm{C}$. to about $100^{\circ} \mathrm{C}$. to produce a vapor mixture of solvent and water. and further comprising the step of transporting the vapor mixture out of the rinse station under vacuum.

7. The method of claim 6. wherein the wash station and the drying station are operated at negative pressure.

8. The method of claim 1. wherein the polyurethane foam is maintained in the rinse station between about 3 minutes and about 15 minutes.

9. The method of claim 1. wherein the temperature of hot air in the drying station does not exceed about $150^{\circ} \mathrm{C}$.

10. The method of claim 9. wherein the organic solvent has a decomposition temperaure and the polyurethane foam has a degradation temperature and the temperature of the hot air in the drying station is maintained at a temperaure that is less than the lower of the decomposition temperature of the solvent or the degradation temperarure of the polyurethane foam.

11. The method of claim 10. wherein the polyurethane foam is maintained in the drying station between about 3 minutes to about 15 minutes.

12. A method of cleaning polyurethane foam. comprising transporting polyurethane foam through a longitudinally extending wash station while altenately soaking the polyurethane foam while immersed in an organic solvent and squeezing solvent from the polyurethane foam a plurality of times by comprising the polyurethane foam as it passes axially through the wash station. conveying the polyure- 35 thane foam from the wash station to a rinse station. transporting the polyurathane foam through a water rinse station. conveying the polyurethane foam from the water rinse station to a drying station. transparting the rinsed polyurethane foam through a longitudinally extending drying station wherein the polyurethane foam is repeatedly squeezed while being exposed to hot air. and continuously removing wet air from the drying station.

13. The method of claim 1. wherein the wash station has 45 a longitudinally extending platen spaced from a path along which the polyurethane foam travels through the wash station. and repeatedly moving the platen toward and away from the polyurethane foam during transportation thereof through the wash station to compress the polyurethane foam a plurality of times during passage of the polyurethane foam through the wash station.

14. The method of claim 13. wherein the polyurethane foam is transported through the wash station on a conveyor beit positioned below the platen.

15. The method of claim 14. wherein an outfeed conveyor is located adjacent the conveyor belt for receiving poiyurethane foam from the conveyor belt after the foam has been compressed a plurality of times by movement of the platen and conveying the polyurethane foam out the organic solyent in the wash station.

16. The method of claim 15. wherein roller assemblies associated with the outfeed conveyor mechanically squeeze solvent from the polyurethane foam until the retained solvent in the polyurethane foam does not exceed about the weight of the polyurethane foam.

\section{8}

17. The method of claim 15. wherein the water in the rinse station is maintained at a temperature in the range of from about $85^{\circ} \mathrm{C}$. to about $100^{\circ} \mathrm{C}$. the wash station and the rinse station being maintained at negative pressure.

18. The method of claim 17. wherein solvent and water evaporated during operation of the rinse station are separated with water being recycied to the rinse station and solvent being recycled to the wash station.

19. The method of claim 17. wherein polyurethane foam is transported through a plurality of axially spaced apart rollers in the drying station alfernately to squeeze and expand the foam.

20. The method of claim 19. wherein the organic solvent. has a decomposition temperature and the polyurethane foam has a degradation temperaure and hot air flow in the drying station is both counter current and cross current to the direction of foam travel.

21. The method of claim 20. wherein the hot air tempera. ture in the drying station is maintained at a temperature that does not exceed the lesser of the decomposition temperature of the solvent or the degradation temperature of the foam.

22. A method of cleaning polyurethane foam having a degradation ternperature. comprising transporting polyurethane foam through a wash station having a bath of organic solvent having a decomposition termperature while alternately soaking the polyurethane foam in a bath of the organic solvent and mechanically squeezing solvent from the polyurethane foam a plurality of times to produce polyurethane foam cleanes than at entry and solvent contaminated with dissolved organic oils and inorganic dirt dispersed in the solvent. removing contaminated organic solvent and transporting same to a cyclone separator to remove inorganic dirt and to an evaporator to separate clean organic solvent. recycling clean organic solvent to the wash station. transporting the polyurethane foam from the wash station through a hot water rinse station. collecting water vapor and organic solvent vapor from the hot water rinse station and separating the constituents to recycte the organie soivent to the wash station and the water to the rinse station. transporting the rinsed polyurethane foam from the rinse station to a dirying station wherein the foam is repeatedly squeezed by passing through rollers to squeeze the foam while being exposed to counter current and cross cument hot air at a temperature less than the lower of the decomposition temperanure of the organic solvent and the degradation temperature of the polyurethane foam and continuously removing wet air from the drying station.

23. The method of claim 22. wherein the organic solvent is perchloroethylene.

so 24. The method of claim 23. wherein the residence time of the polyurethane foam in the wash station is not more than about 15 minutes.

25. The method of claim 23 . wherein the residence time of the polyurethane foam in the rinse station is not more than ss about 15 minutes.

26. The method of claim 23. wherein the residence time of the polyurethane foam in the drying station is not more than about 15 minutes.

27. The method of claim 22 . wherein the polyurethane foam is squeezed between about 20 to about 100 times in each of the wash station and the rinse station and the drying station.

28. A method of cleaning polyurethane foam comprising 65 transporing polyurethane foam from a source thereof through a wash station while alternately soaking the potyurethane foam in a liguid bath of an organic solvent and 


\subsection{2 .432}

9

squeezing solvent from the polyurethane foam a plurality of times. transporting the polyurethane foam through a rinse station. transporting the rinsed polyurethane foam to a drying station wherein the foam is repeatedly squeezed while being exposed to hot air. and continuously removing

wet air from the drying station. wherein said wash station is maintained at negative pressure to prevent escape of solvent vapors to the atmosphere. 


\section{Appendix F Chemistry of the Waste Streams}

This appendix contains the results of the TCLP analysis of the waste streams generated by the continuous foam cleaning Process. Table F.1 gives the results for the solid waste stream, Table F.2 gives the results for the oil waste stream, and Table F.3 gives the results for the waste water stream. The results show that all three waste streams are not hazardous.

Table F.1 Results of the TCLP Analysis of the Solid Waste Generated by the Continuous Foam Cleaning Process

\begin{tabular}{|c|c|c|}
\hline Material & $\begin{array}{c}\text { Concentration } \\
(\mathrm{mg} / \mathrm{L})\end{array}$ & $\begin{array}{c}\text { RCRA Max } \\
\text { (mg/L) }\end{array}$ \\
\hline \multicolumn{3}{|l|}{ Metals: } \\
\hline Arsenic & $<0.05$ & 5 \\
\hline Barium & 2.56 & 100 \\
\hline Cadmium & 0.51 & 1 \\
\hline Chromium & 0.22 & 5 \\
\hline \multicolumn{3}{|l|}{ Organics: } \\
\hline Benzene & $<0.1$ & 0.5 \\
\hline Carbon tetrachloride & $<0.1$ & 0.5 \\
\hline Chlorobenzene & $<1$ & 100 \\
\hline Chlordane & $<0.02$ & 0.03 \\
\hline Chloroform & $<1$ & 6 \\
\hline O Cresol & $<10$ & 200 \\
\hline M Cresol & $<10$ & 200 \\
\hline P Cresol & $<10$ & 200 \\
\hline $2,4 \mathrm{D}$ & $<5$ & 10.0 \\
\hline 1,4 Dichlorobenzene & $<5$ & 7.5 \\
\hline 1,2 Dichlorobenzene & $<0.1$ & 4.3 \\
\hline 1,1 Dichlorobenzene & $<0.1$ & 0.7 \\
\hline 2,4 Dinitrotoluene & $<0.1$ & 0.13 \\
\hline Endrin & $<0.01$ & 0.02 \\
\hline Heptachlor & $<0.004$ & 0.008 \\
\hline Heptachlor epoxide & $<0.004$ & 0.008 \\
\hline
\end{tabular}


Table F.1 (Cont.)

\begin{tabular}{|c|c|c|}
\hline Material & $\begin{array}{c}\text { Concentration } \\
(\mathrm{mg} / \mathrm{L})\end{array}$ & $\begin{array}{c}\text { RCRA Max } \\
\text { (mg/L) }\end{array}$ \\
\hline \multicolumn{3}{|l|}{ Metals: } \\
\hline Lead & 0.96 & 5 \\
\hline Mercury & $<0.002$ & 0.2 \\
\hline Selenium & $<0.05$ & 1 \\
\hline Silver & $<0.2$ & 5 \\
\hline \multicolumn{3}{|l|}{ Organics: } \\
\hline Hexachlorobenzene & $<0.4$ & 0.13 \\
\hline Hexachloroethane & $<2$ & 3 \\
\hline Lindane & $<0.3$ & 0.4 \\
\hline Methyl ethyl ketone & $<10$ & 200 \\
\hline Methoxychlor & $<8$ & 10.0 \\
\hline Nitrobenzene & $<1.5$ & 2 \\
\hline Pentachlorophenol & $<10$ & 100 \\
\hline Pyridine & $<4$ & 5 \\
\hline Tetrachloroethene & $<0.5$ & 0.7 \\
\hline 2,4,5 TP (Silvex) & $<0.8$ & 1.0 \\
\hline Toxaphene & $<0.4$ & 0.5 \\
\hline Trichloroethene & $<0.4$ & 0.5 \\
\hline 2,4,5 Trichlorophenol & $<20$ & 400 \\
\hline 2,4,6 Trichlorophenol & $<1$ & 2 \\
\hline Vinyl chloride & $<0.1$ & 0.2 \\
\hline
\end{tabular}


Table F.2 Results of the TCLP Analysis of the Oil Waste Generated by the Continuous Foam Cleaning Process

\begin{tabular}{|lcc|}
\hline \multicolumn{1}{|c}{ Material } & $\begin{array}{c}\text { Concentration } \\
\text { (mg/L) }\end{array}$ & $\begin{array}{c}\text { RCRA Max } \\
\text { (mg/L) }\end{array}$ \\
\hline Metals: & & \\
Arsenic & & \\
Barium & 0.019 & 5 \\
Cadmium & $<1$ & 100 \\
Total chromium & 0.62 & 1 \\
Hexavalent chromium & $<0.05$ & 5 \\
& $<0.05$ & \\
Organics: & & \\
& & \\
Vinyl chloride & $<0.1$ & 0.2 \\
1,1 Dichloroethene & $<0.1$ & 0.7 \\
Methyl ethyl ketone & $<0.1$ & 200 \\
Chloroform & $<0.1$ & 6 \\
1,2 Dichloroethane & $<0.1$ & 0.5 \\
Carbon tetrachloride & $<0.1$ & 0.5 \\
Benzene & $<0.1$ & 0.5 \\
Trichloroethene & $<0.1$ & 0.5 \\
Tetrachloroethene & 0.161 & 0.7 \\
Chlorobenzene & $<0.1$ & 100 \\
1,4 Dichlorobenzene & $<0.1$ & 7.5 \\
Pyridine & $<0.025$ & \\
\hline
\end{tabular}


Table F.2 (Cont.)

\begin{tabular}{|c|c|c|}
\hline Material & $\begin{array}{c}\text { Concentration } \\
(\mathrm{mg} / \mathrm{L})\end{array}$ & $\begin{array}{l}\text { RCRA Max } \\
\text { (mg/L) }\end{array}$ \\
\hline \multicolumn{3}{|l|}{ Metals: } \\
\hline Lead & 3.88 & 5 \\
\hline Mercury & $<0.005$ & 0.2 \\
\hline Selenium & $<0.05$ & 1 \\
\hline Silver & $<0.05$ & 5 \\
\hline \multicolumn{3}{|l|}{ Organics: } \\
\hline O Cresol & $<0.025$ & 200 \\
\hline M Cresol & $<0.025$ & 200 \\
\hline P Cresol & $<0.025$ & 200 \\
\hline Hexachloroethane & $<0.025$ & 3 \\
\hline Nitrobenzene & $<0.025$ & 2 \\
\hline Hexachlorobutadiene & $<0.025$ & 0.5 \\
\hline $2,4,5$ Trichlorophenol & $<0.025$ & 400 \\
\hline 2,4,6 Trichlorophenol & $<0.025$ & 2 \\
\hline 2,4 Dinitrotoluene & $<0.025$ & 0.13 \\
\hline Hexachlorobenzene & $<0.025$ & 0.13 \\
\hline Pentachlorophenol & $<0.025$ & 100 \\
\hline
\end{tabular}


Table F.3 Results of the TCLP Analysis of the Waste Water Generated by the Continuous Foam Cleaning Process

\begin{tabular}{|c|c|c|}
\hline Material & $\begin{array}{c}\text { Concentration } \\
\text { (mg/L) }\end{array}$ & $\begin{array}{c}\text { RCRA Max } \\
\text { (mg/L) }\end{array}$ \\
\hline \multicolumn{3}{|l|}{ Metals: } \\
\hline Arsenic & $<0.01$ & 5 \\
\hline Barium & $<1$ & 100 \\
\hline Cadmium & $<0.05$ & 1 \\
\hline Total chromium & $<0.05$ & 5 \\
\hline Hexavalent chromium & $<0.05$ & \\
\hline \multicolumn{3}{|l|}{ Organics: } \\
\hline Vinyl chloride & $<0.05$ & 0.2 \\
\hline 1,1 Dichloroethene & 0.136 & 0.7 \\
\hline Methyl ethyl ketone & $<0.05$ & 200 \\
\hline Chloroform & $<0.05$ & 6 \\
\hline 1,2 Dichloroethane & $<0.05$ & 0.5 \\
\hline Carbon tetrachloride & $<0.05$ & 0.5 \\
\hline Benzene & $<0.05$ & 0.5 \\
\hline Trichloroethene & $<0.05$ & 0.5 \\
\hline Tetrachloroethene & $<0.05$ & 0.7 \\
\hline Chlorobenzene & $<0.05$ & 100 \\
\hline 1,4 Dichlorobenzene & $<0.05$ & 7.5 \\
\hline Pyridine & $<0.025$ & 5 \\
\hline
\end{tabular}


Table F.3 (Cont.)

\begin{tabular}{|lcc|}
\hline \multicolumn{1}{|c}{ Material } & $\begin{array}{c}\text { Concentration } \\
\text { (mg/L) }\end{array}$ & $\begin{array}{c}\text { RCRA Max } \\
\text { (mg/L) }\end{array}$ \\
\hline Metals: & & \\
Lead & 0.39 & \\
Mercury & $<0.005$ & 5 \\
Selenium & $<0.05$ & 0.2 \\
Silver & $<0.05$ & 1 \\
& & 5 \\
Organics: & & \\
& & \\
O Cresol & $<0.025$ & \\
M Cresol & $<0.025$ & 200 \\
P Cresol & $<0.025$ & 200 \\
Hexachloroethane & $<0.025$ & 3 \\
Nitrobenzene & $<0.025$ & 2 \\
Hexachlorobutadiene & $<0.025$ & 0.5 \\
2,4,5 Trichlorophenol & $<0.025$ & 400 \\
2,4,6 Trichlorophenol & $<0.025$ & 2 \\
2,4 Dinitrotoluene & $<0.025$ & 0.13 \\
Hexachlorobenzene & $<0.025$ & 0.13 \\
Pentachlorophenol & $<0.025$ & 100 \\
\hline
\end{tabular}

